University of Nebraska - Lincoln

DigitalCommons@University of Nebraska - Lincoln

Brigitte Tenhumberg Papers

Papers in the Biological Sciences

2021

Spectral properties of a non-compact operator in ecology

Matthew Reichenbach

Richard Rebarber

Brigitte Tenhumberg

Follow this and additional works at: https://digitalcommons.unl.edu/bioscitenhumberg

Part of the Animal Sciences Commons, and the Ecology and Evolutionary Biology Commons

This Article is brought to you for free and open access by the Papers in the Biological Sciences at DigitalCommons@University of Nebraska - Lincoln. It has been accepted for inclusion in Brigitte Tenhumberg Papers by an authorized administrator of DigitalCommons@University of Nebraska - Lincoln. 


\title{
Spectral properties of a non-compact operator in ecology
}

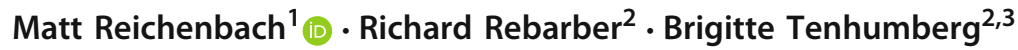

Received: 7 May 2020 / Revised: 22 February 2021 / Accepted: 24 March 2021 / Published online: 13 April 2021 (c) This is a U.S. government work and not under copyright protection in the U.S.; foreign copyright protection may apply 2021

\begin{abstract}
Ecologists have recently used integral projection models (IPMs) to study fish and other animals which continue to grow throughout their lives. Such animals cannot shrink, since they have bony skeletons; a mathematical consequence of this is that the kernel of the integral projection operator $T$ is unbounded, and the operator is not compact. To our knowledge, all theoretical work done on IPMs has assumed the operator is compact, and in particular has a bounded kernel. A priori, it is unclear whether these IPMs have an asymptotic growth rate $\lambda$, or a stable-stage distribution $\psi$. In the case of a compact operator, these quantities are its spectral radius and the associated eigenvector, respectively. Under biologically reasonable assumptions, we prove that the non-compact operators in these IPMs share some important traits with their compact counterparts: the operator $T$ has a unique positive eigenvector $\psi$ corresponding to its spectral radius $\lambda$, this $\lambda$ is strictly greater than the supremum of the modulus of all other spectral values, and for any nonnegative initial population $\varphi_{0}$, there is a $c>0$ such that $T^{n} \varphi_{0} / \lambda^{n} \rightarrow c \cdot \psi$.
\end{abstract}

Keywords Population dynamics · Indeterminate growth · Integral projection models · Positive operators · Essential spectrum · Perron-Frobenius theorem . Measures of non-compactness

Mathematics Subject Classification 47A75 $\cdot 47 \mathrm{G} 10 \cdot 37 \mathrm{~N} 25 \cdot 92 \mathrm{D} 25 \cdot 92 \mathrm{D} 40$

Matt Reichenbach

matt.reichenbach@huskers.unl.edu

1 Geospatial Research Laboratory, US Army Corps of Engineers - Engineer Research and Development Center, 7701 Telegraph Road, Alexandria, VA 22315, USA

2 Department of Mathematics, University of Nebraska-Lincoln, 203 Avery Hall, Lincoln NE68588-0130, USA

3 School of Biological Sciences, University of Nebraska-Lincoln, 402 Manter Hall, Lincoln, NE 68588, USA 


\section{Integral projection models in ecology}

In this paper, we are concerned with operators which arise in integral projection models (or IPMs) describing animal populations in which the individuals exhibit indeterminate growth; that is, when individuals continue to grow throughout their lives. The operators in IPMs are not projections in the mathematical sense of the word; the term projection comes from the fact that these models "project" a current population size and structure into the future. We will exclusively use the term IPM hereafter to avoid confusion.

IPMs are discrete-time, stage-structured models introduced by Easterling (1998) and Ellner and Rees (2006); they generalize Leslie matrices (see for instance the book by Caswell (2001)) by allowing for the structure variable to take on values in a continuum. Hence, IPMs are appropriate when vital rates depend on a continuous variable, such as the length or biomass of an individual. Briggs et al. (2010) give a gentle introduction to constructing IPMs, whereas Ellner et al. (2016) give a more detailed overview.

The IPMs we consider in this paper are given by an integral operator $T: L^{1} \rightarrow L^{1}$ where

$$
\varphi_{t+1}(y)=\left(T \varphi_{t}\right)(y):=\int_{L}^{U} k(y, x) \varphi_{t}(x) d x .
$$

Here, $\varphi_{t}(x)$ is the population density in stage $x$ at time $t$, the nonnegative kernel function $k(y, x)$ describes how the distribution of individuals in stage $x$ contributes to the individuals in stage $y$ in the next time step, and $L$ and $U$ are the lower- and upper-limits of the structure variable, respectively. In this paper, we consider IPMs such that the kernel function $k(y, x)$ can be decomposed as

$$
s(x) g(y, x)+b(y) f(x),
$$

where $s(x)$ is the survival probability of an individual in stage $x, g(y, x)$ gives the probability that a stage $x$ individual grows to a stage $y$ individual in one time step, $b(y)$ is the size distribution of newborns, and $f(x)$ gives the expected number of offspring that an average individual in stage $x$ will produce in one time step. Together, the function $s(x) g(y, x)$ is known as the survival and growth subkernel, and $b(y) f(x)$ as the fecundity subkernel. In practice, these functions are usually determined by fitting appropriate probability distributions to population data.

IPMs have found wide use in the biological sciences (Ellner et al. (2016) give a good overview). In the case that the kernel function $k(y, x)$ is continuous and positive on the square $[L, U]^{2}$, Ellner and Rees (2006) proved (among other things) that the associated IPM operator $T$ has its spectral radius as an eigenvalue, and the associated eigenvector is nonnegative. In biological terms, the spectral radius of the operator $T$ is the asymptotic growth rate of the population, which we will denote by $\lambda$, and the associated eigenvector $\psi$ is the stable stage distribution. The eigenvector $\psi$ is important in conservation biology, because by comparing the stable stage distribution with a population distribution in the field, a biologist can determine if the field population has reached the steady state. 
Ellner and Rees (2006) assumed that the kernel function $k(y, x)$ is bounded, which implies that $T$ is a compact operator on the relevant function space. An IPM with a compact operator is easier to work with because compact operators can be uniformly approximated by matrices. Ecologists can thus estimate the asymptotic growth rate of a population by approximating the infinite-dimensional operator with large matrices; the leading eigenvalue of these large matrices will be a good approximation of the asymptotic growth rate of the population, as predicted by the IPM.

The appropriate choice for the structure variable depends on the ecology of the species concerned, or what data ecologists can collect. Some common examples of structure variables in IPMs are animal biomass, stem diameter of plants, the proportion of tissue infected by a disease, and the length or height of individuals. However, this choice has mathematical consequences: if individuals can decrease in size from one time step to the next, the IPM operator $T$ will be compact; if individuals cannot decrease in size, we prove in Sect. 6 that the $T$ will not be compact. In the former case, the results of Ellner and Rees (2006) apply to the operator $T$.

For structure variables that cannot decrease over time, i.e., when the probability of shrinkage is zero, the growth subkernel $g(y, x)$ is unbounded. To get an intuitive idea for why this is the case, suppose that $G$ is the integral operator with kernel $g(y, x)$; this operator models the somatic growth of individuals over one time step. If individuals cannot shrink, and must continue to grow, then applying $G$ repeatedly yields a population dominated by individuals near the maximum body size. Since $g(y, x)$ does not incorporate mortality, the growth subkernel $g(y, x)$ must capture the growth of an increasingly concentrated population. Hence, $g(y, x)$ will be unbounded near the point $(U, U)$, where $U$ is the maximum body size. If the function $g(y, x)$ is unbounded, then the full IPM kernel $k(y, x)$ will be as well. In general, it is possible for compact integral operators to have unbounded kernels; if this were the case for an IPM operator $T$, then the results proved by Ellner and Rees (2006) would still apply. But in this paper, we will show that assuming individuals do not shrink implies not only that the kernel $k(y, x)$ is unbounded, but also that the associated integral operator is not compact. This means that the results proved by Ellner and Rees (2006) do not apply to these populations, thus making it unclear whether IPMs with non-compact operators have an asymptotic growth rate or a stable stage distribution.

Most IPMs in the literature have compact operators. Examples include those which model plant species that can shrink over time in poor growing conditions (Childs et al. 2003; Eager et al. 2013; Hegland et al. 2010; Jacquemyn et al. 2010; Miller et al. 2012; Tenhumberg et al. 2015; Rees and Rose 2002). Additionally, Childs et al. (2011), Ellner et al. (2016), Coulson et al. (2011) used the biomass of sheep and wolf individuals as their structure variable, which can also decrease in poor environmental conditions. Bruno et al. (2011) were interested in the proportion of a coral covered by a fungal infection, which can decrease over the course of a time step.

Alternatively, some authors have used the length of fish and mollusks as structure variables, namely Aalto et al. (2019), Ohlberger et al. (2020), Stubberud et al. (2019), Vindenes et al. (2014) and Vindenes and Langangen (2015), presumably because these were the only data available for parameterizing their IPM models. Since length cannot decrease from one time step to the next, their IPM operators are non-compact. 
In this paper, we show that biologically relevant properties, such as the existence of an asymptotic growth rate and a stable stage distribution for the population, still hold for the non-compact operator T. This allows ecologists to gain biological insight from IPMs in which individuals cannot shrink between time steps. As an example, we show that our results, combined with an argument given in Sect. 10, show that an IPM for northern pike has an asymptotic growth rate larger than 1; that is, the model predicts the population grows in the long-run.

\section{Mathematical motivations}

IPMs are generalizations of matrix population models of the form

$$
\mathbf{n}_{t+1}=A \mathbf{n}_{t}, \quad \mathbf{n}_{0} \in \mathbb{R}^{n},
$$

where $A$ is a matrix, and $\mathbf{n}_{0}$ a vector, both with non-negative entries. The relevant spectral properties of the matrix $A$ in these models are guaranteed by the PerronFrobenius theorem (see the book by Caswell (2001)). Among other things, population matrices have the following three properties:

(1) the spectral radius $r(A)$ is positive, and is an eigenvalue for $A$. The right and left eigenvectors $\mathbf{v}, \mathbf{v}^{*}$ associated to $r(A)$ are the only eigenvectors of $A$ which can be normalized to have all positive entries;

(2) the matrix A has a "spectral gap", meaning that

$$
\max \{\sigma(\mathrm{A}) \backslash\{\lambda\}\}<r(\mathrm{~A})
$$

(3) for any $\mathbf{n}_{0} \in \mathbb{R}^{n}$ with nonnegative entries, and $\lambda=r(\mathrm{~A})$ the spectral radius of $\mathrm{A}$ associated to the right and left eigenvectors $\mathbf{v}, \mathbf{v}^{*}$, we have $\left\langle\mathbf{n}_{0}, \mathbf{v}^{*}\right\rangle>0$, and

$$
\lim _{k \rightarrow \infty} \frac{\mathrm{A}^{k} \mathbf{n}_{0}}{\lambda^{k}}=\left\langle\mathbf{n}_{0}, \mathbf{v}^{*}\right\rangle \mathbf{v},
$$

where $\langle\cdot, \cdot\rangle$ denotes the dot product in $\mathbb{R}^{n}$.

In biological terms, property (3) means that the population has a long-term growth rate of $\lambda=r(\mathrm{~A})$, and the vector $\mathbf{v}$ is known as the stable stage distribution. The vector $\mathbf{v}$ gives the relative proportions of each stage in the long-term population, or in other words, $\mathbf{v}$ captures the proportions one would expect to see in the population absent any external perturbations.

Krein and Rutman (1950) showed that certain compact operators have property (1), and many authors have since obtained generalizations of their theorem to wider classes of operators (for example, see the papers by Anselone and Lee (1974), Bonsall (1958), Edmunds et al. (1972), Karlin (1959), Krasnosel'skij et al. (1989), Lubben et al. (2009), Marek (1967), Marek (1970), Raghavan (1965), and Schaefer (1960)). Various authors have also obtained results like (2) for a wider class of operators (see e.g. Chapter 12 of the book by Krasnosel'skij et al. (1989), and the references cited therein). In an 
appendix to the the paper by Ellner and Rees (2006), the authors showed that certain compact operators arising from IPMs in mathematical ecology satisfy properties (1), (2), and (3).

In this paper, we consider a class of operators which come from IPMs recently constructed by mathematical ecologists, but for which the results proved by Ellner and Rees (2006) do not apply. Specifically, the operators we consider are not compact. Out of the papers listed above, (Bonsall 1958; Edmunds et al. 1972; Karlin 1959; Krasnosel'skij et al. 1989; Marek 1967, 1970; Raghavan 1965; Schaefer 1960) studied operators $T: X \rightarrow X$ which are not necessarily compact. Instead, the authors impose topological conditions on the space $X$, or specific conditions on the operator $T$, in order to prove their results.

For our purposes, the results proved by Marek (1970), Sawashima (1964), and Schaefer (1960) will be useful to us in showing that the operators we consider have properties (1)-(3) of the Perron-Frobenius theorem. Specifically, we will show that the operator $T: L^{1} \rightarrow L^{1}$ is not compact, that it is strictly nonsupporting, and that its spectral radius $r(T)$ is a pole of the resolvent operator $R(z, T)$. We will prove these facts in Sects. 6, 7, and 8 respectively. In Sect. 9, we will show that the non-compact operator $T$ has properties (1)-(3) above. This means that IPMs with non-compact operators of the form we consider have the theoretical properties one would want in a population model. We note that IPMs are discrete-time models, but results like (1)-(3) above are known for continuous-time models as well; see Chapters 8-10 in the book by Clément et al. (1987).

\section{Definition of the IPM operator and its components}

For functional analysis concepts and notation, we follow the book by Conway (1994). All integrals will be with respect to the Lebesgue measure $\mu$, and "a.e" means "almosteverywhere" with respect to $\mu$. Let $\Omega:=[L, U]$ denote a closed and bounded interval of $\mathbb{R}$. In practice, $L, U$ will be positive values denoting the lower and upper limits for the structure variable, respectively.

We will use the notation $L^{1}:=L^{1}(\Omega)$ to denote the Banach space of integrable real-valued functions with norm

$$
\|\varphi\|_{1}:=\int_{L}^{U}|\varphi(t)| d t
$$

The space $L^{1}$ is the natural space to work in for biological applications, because the norm $\|\varphi\|_{1}$ of the nonnegative population vector $\varphi$ gives the total population. We will also make use of the space $L^{\infty}=L^{\infty}(\Omega)$, which is the space of Lebesgue-measurable, essentially bounded functions with norm

$$
\|h\|_{\infty}:=\operatorname{ess} \sup \{|h(t)| \mid t \in \Omega\}
$$


We study integral operators $T: L^{1} \rightarrow L^{1}$ whose kernels take the form

$$
k(y, x)=s(x) g(y, x)+b(y) f(x) .
$$

Here, we will assume that the function $s(x)$ is continuous, increasing, and positive on $\Omega$, with

$$
\sup _{x \in \Omega}\{s(x)\}<1
$$

This means that each individual has a chance of dying during each time step, and the positivity assumption means that any individual has a chance of surviving. We will assume that $b(y)$ is the offspring distribution, bounded almost-everywhere in $\Omega$ and positive almost-everywhere in the set $\left[L, x_{b}\right]$, where we allow (but do not require) that $b(y)$ can be zero for all $y>x_{b}$. In that case, $x_{b}$ is the largest size that an individual can attain in one time step after birth.

Additionally, we suppose that there is some "size of maturity" $x^{\prime} \in[L, U)$ such that the fecundity function $f(x)$ is almost-everywhere bounded away from zero for $x \geq x^{\prime}$. We have been unable to find an IPM in which these assumptions on $f(x)$ are not satisfied, and our results apply just as well when $f(x)>0$ throughout $\Omega$ (in this case, one can take $x^{\prime}=L$ ).

Taken together, these assumptions on $s(x), b(y)$, and $f(x)$ imply the existence of positive numbers, $s_{0}, s_{1}, b_{1}$, and $f_{0}, f_{1}$ such that

$$
\begin{aligned}
& 0<s_{0} \leq s(x) \leq s_{1}<1, \quad \text { for almost every } x \in \Omega, \\
& 0<b(y) \leq b_{1}<\infty, \quad \text { for almost every } y \in \Omega, \\
& 0<f_{0} \leq f(x) \leq f_{1}<\infty, \quad \text { for almost every } x \in\left[x^{\prime}, U\right]
\end{aligned}
$$

It will be convenient to assume that $s_{1}, b_{1}, f_{1}$ are the least such values, and that $f_{0}$ is the greatest such value.

We assume that $g(y, x)$ is nonnegative on $[L, U)^{2}$ (note the open right endpoint), and also that for each $x \in[L, U)$,

$$
\int_{L}^{U} g(y, x) d y=1 .
$$

The assumption (3.5) means that $g(y, x)$ is a probability distribution for each fixed $x \in[L, U)$. In biological language, this means that a size $x$ individual will have size $y \in[L, U)$ in the following time step with probability $g(y, x)$. We will often refer to $g(y, x)$ as the growth subkernel of $T$.

Of particular interest to us in this paper are operators with a kernel of the form (3.1) such that

$$
g(y, x)=0, \quad \text { whenever } y<x,
$$


and in this case we will say that $g(y, x)$ is "zero below the diagonal". When the operator $T$ models a stage-structured population such that individuals cannot move to lower stages (for example, when $\Omega$ is a set of possible lengths, and individuals exhibit indeterminate growth), $g(y, x)$ satisfies (3.6). Note that we do not require $g(y, x)$ to be continuous, so it may be positive for $y=x$ and still satisfy (3.6). This allows $g(\cdot, x)$ to be any probability distribution which is appropriate for the population, so long as (3.5)-(3.12) are all satisfied.

Taken together, assumptions (3.5) and (3.6) imply that $g(y, x)$ is unbounded in any neighborhood of the point $(U, U) \in \mathbb{R}^{2}$, which is why we assume $g(y, x)$ is defined on $[L, U)^{2}$, rather than $[L, U]^{2}$.

Example 3.1 We have included Fig. 1 below as an example of an unbounded growth kernel from Vindenes et al. (2014); they include an extra parameter $z$ for temperature, which we set to $10.34{ }^{\circ} \mathrm{C}$, the mean of the time series they consider. The function in Fig. 1 is given by

$$
g(y, x)=\left\{\begin{array}{ll}
\frac{\rho(y, x)}{\int_{x}^{U} \rho(y, x) d y}, & y>x, \\
0, & y \leq x
\end{array},\right.
$$

where

$$
\begin{aligned}
\rho(y, x) & :=\frac{1}{\sqrt{2 \pi}(y-x) v(x)} \exp \left(-\frac{(\ln (y-x)-\mu(x))^{2}}{2 v(x)}\right) \\
\mu(x) & :=\log \left(\frac{(m(x)-x)^{2}}{\sqrt{(m(x)-x)^{2}+\sigma(x)^{2}}}\right) \\
v(x) & :=\log \left(1+\frac{\sigma(x)^{2}}{(m(x)-x)^{2}}\right) .
\end{aligned}
$$

Equation 3.7 is the lognormal probability density function for the growth increment $(y-x)$. In Eqs. (3.8) and (3.9), the function $m(x)$ is the "average expected size" function (which is usually fit to population data), and $\sigma(x)$ is the standard deviation of sizes at size $x$. Also, note that $m(x)$ and $\sigma(x)$ are functions of the size $x$ on a linear scale, hence why the conversions (3.8) and (3.9) are necessary.

In Fig. 1, we left the surface unshaded in the region $y<x$, to indicate that $g(y, x)=$ 0 there. This is the way to incorporate the biological assumption that individuals cannot transition to a smaller size; i.e., they cannot "shrink". Note that the plot becomes unbounded in a neighborhood of the point $(U, U)$, where $U$ is the maximum size.

In Ellner and Rees (2006), the authors assumed that their IPM kernel was strictly positive and continuous, and hence bounded away from zero in the square $[L, U]^{2}$. We cannot make this same assumption, because we allow the component functions $g(y, x), b(y)$, and $f(x)$ to possibly be zero in sets of positive measure. Hence, we will need further assumptions to prove similar results as Ellner and Rees (2006) did; we will denote these assumptions by $(\mathbf{M}),(\mathbf{R})$, and $(\mathbf{S})$ : 


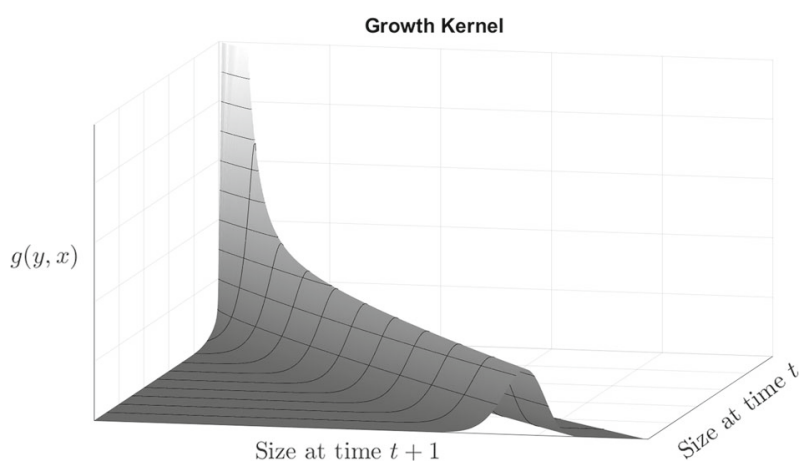

Fig. 1 The growth kernel $g(y, x)$ for the northern pike IPM

(M) there is a continuous, strictly increasing function $\eta:[L, U] \rightarrow[L, U]$ such that $\eta(U)=U, \eta(x)>x$ for all $x \in[L, U)$, and

$$
\begin{aligned}
& \int_{\eta(x)}^{U} g(y, x) d y>0, \quad \text { for a.e. } x \in \Omega, \\
& \int_{L}^{\eta^{-1}(y)} g(y, x) d x>0, \text { for a.e. } y>\eta(L), \\
& g(y, x)>0, \quad \text { for a.e. }(y, x) \text { such that } x<y<\eta(x) \text {; }
\end{aligned}
$$

(R) there exists an $\varepsilon_{1}>0$ and a closed rectangle $R \subseteq[L, U)^{2}$ of the form

$$
R:=\left[U-\varepsilon_{1}, U\right] \times\left[t_{1}, t_{2}\right],
$$

such that for $L \leq t_{1} \leq t_{2} \leq y$, and $g(y, x)>0$ almost-everywhere in $R$;

(S) there is some $\varepsilon_{2}>0$ such that $s(x) \equiv s_{1}$ for $x \in\left[U-\varepsilon_{2}, U\right]$.

In Example 3.1 above, one can check that $g(y, x)$, with the function $\mu(x)$ taken to be $\eta$, satisfies assumption (M). For IPMs which do not require a conversion to log-scales for length, the average-expected size $m(x)$ satisfies assumption $(\mathbf{M})$ in all cases we are aware of. Assumption $(\mathbf{R})$ looks onerous, but if a growth kernel $g(y, x)$ is obtained by fitting positive probability distributions to data, it will satisfy assumption (R).

Assumption (S) is a technical condition we need to prove a result, and the IPM operators in Ohlberger et al. (2020), Stubberud et al. (2019), Vindenes et al. (2014), Vindenes and Langangen (2015) all satisfy it. The biological interpretation of (S) is that individuals near the maximum size $x=U$ have maximal survival chances. Vindenes et al. (2014) note that there are usually very few individuals in a population near the maximum size, so they consider it a reasonable simplification to assume survival is constant near $x=U$. However, they also note that in many cases assumption (S) likely won't hold. For example, the survival function $s(x)$ may decrease near the maximum size when large individuals experience senescence (as they are likely to be older), or as an artifact of data collection. To this latter point, Vindenes et al. (2014) suggest that 
if an IPM for fish is parameterized using data from a mark-recapture survey which uses gillnets, the largest fish may not be caught and will thus appear to have a lower survival probability. Additionally, $s(x)$ could decrease at larger sizes if the population is subject to size-specific harvesting, for example when anglers prefer big fish. Due to these considerations, we hope in the future to remove assumption $(\mathbf{S})$, and also the assumption that $s(x)$ is increasing, so that the results of this paper can be applied as widely as possible.

For notational convenience, we will sometimes write $T: L^{1} \rightarrow L^{1}$ in terms of its components

$$
T=G S+b F
$$

where $G: L^{1} \rightarrow L^{1}$ is the integral operator with kernel $g(y, x), S: L^{1} \rightarrow L^{1}$ is multiplication by $s(x), F: L^{1} \rightarrow \mathbb{R}$ is the fecundity functional defined by

$$
\varphi \mapsto \int_{L}^{U} f(t) \varphi(t) d t
$$

and $b$ is the offspring distribution. We will call $G$ the growth operator, the composition $G S$ as the "growth and survival" operator, and $b F$ the "fecundity" operator.

\section{Mathematical preliminaries}

Given a Banach space $X$, we denote the space of continuous linear functionals on $X$ as $X^{*}$; the space $X^{*}$ is known as the Banach dual space of $X$. In this paper, we will only consider the case where $X=L^{1}$, in which case $X^{*}=L^{\infty}$, and the functionals are represented by some $h \in L^{\infty}$ acting by integration on elements in $L^{1}$. We will use the inner-product notation $\langle\varphi, h\rangle$ to denote this action; that is, for $\varphi \in L^{1}$ and $h \in L^{\infty}$, we define

$$
\langle\varphi, h\rangle:=\int_{L}^{U} \varphi(t) h(t) d t .
$$

We will sometimes abuse terminology by referring to the element " $h$ " as a functional, but it should be clear we mean it represents a functional given by (4.1).

Given a linear operator $T: X \rightarrow Y$ between normed vector spaces $X$ and $Y$, we denote the operator norm of $T$ to be the quantity

$$
\|T\|:=\sup \left\{\|T \varphi\|_{Y} ; \text { where }\|\varphi\|_{X}=1\right\}
$$

where the subscripts denote which space the norm is taken in. If this operator norm is finite, we say that $T$ is a bounded operator. The Banach adjoint of $T$, denoted $T^{*}: X^{*} \rightarrow X^{*}$, is the unique operator such that

$$
\langle T \varphi, h\rangle=\left\langle\varphi, T^{*} h\right\rangle
$$


for all $x \in X$ and $h \in X^{*}$.

For a linear operator $T: X \rightarrow X$, with $X$ a vector space over $\mathbb{C}$, the spectrum of $T$ is the set

$$
\sigma(T):=\{z \in \mathbb{C} \mid z I-T \text { is not boundedly invertible }\}
$$

Here, $I$ is the identity operator. Additionally, we denote the spectral radius of the operator $T$ by $r(T)$, where

$$
r(T):=\sup \{|z| ; \text { where } z \in \sigma(T)\}
$$

The peripheral spectrum $\sigma_{p}(T)$ are those $z \in \sigma(T)$ such that $|z|=r(T)$.

Another subset of $\sigma(T)$ which will be useful to us is known as the essential spectrum; we will denote this subset by $\sigma_{e}(T)$. There many definitions of the essential spectrum in the literature, but we follow that given in the papers by Browder (1961) and Edmunds et al. (1972):

Definition 4.1 The essential spectrum $\sigma_{e}(T)$ of an operator $T$ is the collection of complex numbers $z \in \sigma(T)$ such that at least one of the following conditions holds:

(1) the range of $(z I-T)$ is not closed;

(2) $z$ is a limit point of $\sigma(T)$;

(3) $\cup_{n=1}^{\infty} \operatorname{ker}(z I-T)^{n}$ is infinite-dimensional,

where $\operatorname{ker}(\cdot)$ denotes the kernel of its argument.

We also make use of the essential spectral radius of the operator $T$, which we denote $r_{e}(T)$, and which is defined analogously to the ordinary spectral radius:

$$
r_{e}(T):=\sup \left\{|z| \mid z \in \sigma_{e}(T)\right\}
$$

For the other common definitions of the essential spectrum, each has the same essential spectral radius, a fact proved by Edmunds and Evans (1987).

Note that the operators in IPMs are naturally operators on a real vector space; in order to talk about the spectrum of an operator $T: X \rightarrow X$, with $X$ a vector space over $\mathbb{R}$, we define the complexifications of $T$ and $X$, denoted $T_{c}$ and $X_{c}$, where

$$
X_{c}:=X \oplus i X
$$

and $T_{c}: X_{c} \rightarrow X_{c}$ is the linear operator such that

$$
T_{c}\left(\varphi_{1}+i \varphi_{2}\right):=T\left(\varphi_{1}\right)+i T\left(\varphi_{2}\right) .
$$

When we refer to "the spectrum of $T$ ", where $T$ is an operator on a real vector space, we actually mean the spectrum of its complexification $T_{c}$. One can show that $X_{c}$ is 
a Banach space over $\mathbb{C}$, with addition and scalar multiplication defined in the natural way, and with the norm $\|\cdot\|_{c}$ defined by

$\left\|\varphi_{1}+i \varphi_{2}\right\|_{c}:=\frac{1}{\sqrt{2}} \cdot \sup _{0 \leq \theta<2 \pi}\left(\left\|\cos (\theta) \varphi_{1}-\sin (\theta) \varphi_{2}\right\|+\left\|\sin (\theta) \varphi_{1}+\cos (\theta) \varphi_{2}\right\|\right)$

The complexification $T_{c}$ is linear and bounded if and only if $T$ is also linear and bounded. Additionally, the norms of $T$ and $T_{c}$ coincide:

$$
\|T\|=\left\|T_{c}\right\|_{c} .
$$

For more information concerning complexifications of real vector spaces and operators, see the paper by Edmunds et al. (1972).

To compute spectral radii, we will make use of Gelfand's formula, which is the statement that

$$
r(T)=\lim _{n \rightarrow \infty}\|T\|^{1 / n}
$$

The resolvent of $T$ is the function $R(z, T):=(z I-T)^{-1}$, which is well-defined in the resolvent set $\rho(T):=\mathbb{C} \backslash \sigma(T)$. It turns out that $R(z, T)$ is a holomorphic function in $\rho(T)$, and in the case that $|z|>r(T)$, we can write $R(z, T)$ as a so-called Neumann series given by

$$
R(z, T)=\sum_{k=0}^{\infty} \frac{T^{k}}{z^{k+1}}
$$

In order to study the essential spectrum $\sigma_{e}(T)$, we will make use of the ball measure of non-compactness, or ball MNC for short. Some authors also use the term Hausdorff $M N C$. We follow the definitions, terminology, and results given by Akhmerov et al. (1992):

Definition 4.2 The ball measure of non-compactness of a subset $V$ of the vector space $X$, denoted $\beta(V)$, is given by

$$
\beta(V):=\inf \{r>0 \mid V \text { can be covered by finitely many balls of radius } r\} .
$$

Clearly $0 \leq \beta(V) \leq \infty$; other properties of $\beta(\cdot)$ which will be useful to us are:

(1) $\beta(V)=0$ if and only if $V$ is pre-compact (that is, if and only if the closure of $V$ is compact);

(2) For the set

$$
V+W:=\{v+w \mid v \in V, w \in W\}
$$

we have

$$
\beta(V+W) \leq \beta(V)+\beta(W)
$$


for all $V, W \subseteq X$

(3) $V_{1} \subseteq V_{2}$ implies that $\beta\left(V_{1}\right) \leq \beta\left(V_{2}\right)$;

(4) $\beta(\lambda V)=|\lambda| \beta(V)$ for each $\lambda \in \mathbb{C}$;

(5) For any point $x_{0} \in X$, we have $\beta\left(V+x_{0}\right)=\beta(V)$.

For further useful properties that $\beta$ satisfies, see the book by Akhmerov et al. (1992). There are other commonly used MNC's, but the ball-MNC $\beta$ is especially useful for us because there is a formula for $\beta(V)$ when $V \subseteq L^{p}(\mathbb{R}), 1 \leq p \leq \infty$. Let $X$ be one of these spaces, and $V \subseteq X$ any subset. Then we have that

$$
\beta(V)=\frac{1}{2} \lim _{\delta \rightarrow 0} \sup _{\varphi \in V} \sup _{0<\tau \leq \delta}\left\|\varphi-\varphi_{\tau}\right\|_{X}
$$

where $\varphi_{\tau}(t):=\varphi(t+\tau)$.

We note that the domain of the functions we consider is $\Omega=[L, U]$, but we can apply (4.7) by extending their domain to all of $\mathbb{R}$ by setting $\varphi(x)=0$ for $x$ outside $\Omega$, and for every $\varphi \in L^{1}(\Omega)$.

There is a formula for $r_{e}(T)$, due to Nussbaum (1970), which makes use of the ball-MNC $\beta$. Letting $\mathscr{U} \subseteq X$ denote the unit ball in the space $X$, and writing $\beta(T):=$ $\beta(T(\mathscr{U}))$, we have that

$$
r_{e}(T)=\lim _{n \rightarrow \infty} \beta\left(T^{n}\right)^{1 / n}
$$

Note the similarity between this formula for the essential spectral radius, and Gelfand's formula (4.5) for the ordinary spectral radius. Using the formulas (4.7) and (4.8) together, we will be able to compute the essential spectral radius of the operator $T: L^{1} \rightarrow L^{1}$ in Sect. 8.

The operators we study in this paper are examples of "positive" operators, which means that they are invariant on a cone $K$ in a Banach space $X$. We follow the book by Krasnosel'skij et al. (1989) for definitions and theorems regarding cones.

Definition 4.3 A closed convex set $K$ of the real Banach space $X$ is called a cone if the following conditions hold:

(1) for any $x \in K$ and $a \geq 0$, the element $a x$ is in $K$,

(2) for any pair $x, y \in K$, the element $x+y$ is in $K$, and

(3) $K \cap-K=\{0\}$.

Defined in this way, we get a partial ordering on the cone $K$ : for two elements $x$, $y \in K$, we say that $x \leq y$ if and only if $y-x \in K$.

It is straightforward to check that the collections of nonnegative a.e. functions in $L^{1}$ and $L^{\infty}$ are cones; we refer to these as the standard cones in their respective spaces.

Given a cone $K$, we will also make use of its dual cone:

Definition 4.4 Suppose that $X$ is a Banach space with cone $K$, and let $X^{*}$ be the Banach dual space of $X$. The dual cone of $K$, denoted $K^{*} \subseteq X^{*}$, is the collection of all continuous linear functionals $h$ such that $h(x) \geq 0$, for all $x \in K$. 
It is a straightforward exercise to show that if $K$ is the standard cone in $L^{1}$, its dual cone $K^{*}$ is the standard cone in $L^{\infty}$. For the next definition, we use the notation

$$
K-K=\{x-y \mid x, y \in K\} .
$$

Definition 4.5 An operator $T: X \rightarrow X$, (possibly nonlinear), with $X$ a real Banach space, is called positive with respect to a cone $K \subseteq X$ if $T(K) \subseteq K$.

This definition yields a partial order on the set of positive operators; if $T_{1}, T_{2}$ are positive operators, we say that $T_{1} \leq T_{2}$ if $T_{2}-T_{1}$ is a positive operator.

We will sometimes call an operator simply "positive", and drop references to the particular cone $K$, as we are only concerned with the standard cones $K \subseteq L^{1}$ and $K^{*} \subseteq L^{\infty}$. Hereafter, when we write $K$ and $K^{*}$, we mean the standard cones in $L^{1}$, $L^{\infty}$ respectively.

Example 4.1 Supposing it is well-defined, the integral operator $T: L^{1} \rightarrow L^{1}$ of the form

$$
(T \varphi)(y):=\int_{L}^{U} k(y, x) \varphi(x) d x
$$

is an example of a positive operator with the respect to $K$, whenever $k(y, x) \geq 0$ almost-everywhere. Additionally, the Banach dual $T^{*}: L^{\infty} \rightarrow L^{\infty}$ is also a positive operator in that it maps $K^{*}$ into $K^{*}$, and is given by

$$
\left(T^{*} \varphi^{*}\right)(x)=\int_{L}^{U} k(y, x) \varphi^{*}(y) d y .
$$

That is, the Banach adjoint of an integral operator is obtained by "transposing" the kernel function, i.e., by integrating with respect to $y$ instead of $x$.

In Sect. 7, we will show that the IPM operator in this paper is strictly nonsupporting, which is a concept introduced by Sawashima (1964), and further elaborated in Niiro and Sawashima (1966a,b), and Marek (1970). We follow the terminology of Marek (1970) on this topic:

Definition 4.6 Suppose $T$ is a positive operator with respect to the cone $K$, and suppose that $\varphi \in K, \varphi^{*} \in K^{*}$ are both nonzero.

(1) $T$ is called nonsupporting if for every pair $\varphi, \varphi^{*}$ there is a positive integer $p=$ $p\left(\varphi, \varphi^{*}\right)$ such that $\left\langle T^{n} \varphi, \varphi^{*}\right\rangle>0$ for every $n \geq p$.

(2) $T$ is called strictly nonsupporting if for every pair $\varphi, \varphi^{*}$ there is a positive integer $p=p(\varphi)$ such that $\left\langle T^{n} \varphi, \varphi^{*}\right\rangle>0$ for every $n \geq p$.

Note if $T$ is strictly nonsupporting, it is also nonsupporting.

We will also make use of the following concepts in proving our main results:

Definition 4.7 Given a cone $K$, an element $\varphi \in K$ is called quasi-interior if $\left\langle\varphi, \varphi^{*}\right\rangle>$ 0 for all nonzero $\varphi^{*} \in K^{*}$. 
The related notion for the dual cone is the following:

Definition 4.8 Given a cone $K$, an element $\varphi^{*} \in K^{*}$ is called strictly positive if $\left\langle\varphi, \varphi^{*}\right\rangle>0$ for all nonzero $\varphi \in K$.

\section{General results}

In this section, we will prove some fundamental facts about the operator $T: L^{1} \rightarrow L^{1}$ which will be useful in demonstrating many of the results that follow. In particular, at the end of Sect. 6 we will prove that no power $T^{k}$ is weakly compact (and in particular not compact) under the assumption (3.6). This indicates that we will not be able to use the Krein-Rutman theorem, or its generalizations given in the book by Krasnosel'skij et al. (1989), to obtain results about the spectral properties of $T$.

Lemma 5.1 Let $G: L^{1} \rightarrow L^{1}$ be the growth operator, and consider the standard cone $K \subseteq L^{1}$. Then for any $\varphi \in K$, we have that $\|G \varphi\|_{1}=\|\varphi\|_{1}$.

Proof This is a quick application of Fubini-Tonell:

$$
\|G \varphi\|_{1}=\int_{L}^{U} \int_{L}^{U} g(y, x) \varphi(x) d x=\int_{L}^{U} \varphi(x) \underbrace{\int_{L}^{U} g(y, x) d x}_{=1, \text { a.e. } x} d y=\|\varphi\|_{1}
$$

where the last equality comes from the fact that $\varphi \geq 0$.

Corollary 5.1 For each $k \geq 1$, and $\varphi \in K \subseteq L^{1}$, we have $\left\|G^{k} \varphi\right\|_{1}=\|\varphi\|_{1}$.

We mentioned earlier that the kernel function $g(y, x)$, when zero below the diagonal, models the growth of individuals who cannot shrink; we make this intuition rigorous in the next lemma:

Lemma 5.2 Suppose that $\varphi \in K \subseteq L^{1}$ is such that for some $a \in[L, U), \varphi(x)=0$ whenever $x<a$. Then $(G \varphi)(y)=0$ whenever $y<a$ as well.

Proof Take some $\varphi \in K$ satisfying the properties above. Fixing an arbitrary $y<a$, we have that

$$
(G \varphi)(y)=\int_{L}^{U} g(y, x) \varphi(x) d x=\int_{a}^{U} g(y, x) \varphi(x) d x
$$

since $\varphi(x)=0$ for $x<a$. Because $y<a$ and $g(y, x)=0$ whenever $y<x$, we conclude that the second integral above is equal to zero. Since the choice of $y<a$ was arbitrary, we have that $(G \varphi)(y)=0$ for any $y<a$.

With this result, we immediately obtain the corollary

Corollary 5.2 Suppose $\varphi \in K$ and $a \in[L, U)$ are as in Lemma 5.2. For any $k \geq 1$, we have $\left(G^{k} \varphi\right)(y)=0$ whenever $y<a$ as well. 
Lemma 5.3 Let $\eta(x)$ be the continuous and increasing function described in $(\boldsymbol{M})$. For any $x \in[L, U]$, we have that $\eta^{n}(x) \rightarrow U$ as $n \rightarrow \infty$, where

$$
\eta^{n}(x):=\eta\left(\eta^{n-1}(x)\right), \text { and } \eta^{0}(x):=x
$$

Proof The result is immediate for $x=U$, so suppose that $L \leq x<U$. Since $\eta$ is strictly increasing, we have that

$$
x<\eta(x)<\eta^{2}(x)<\cdots \eta^{n}(x)<\cdots \leq U
$$

for every $n$. Since $\left\{\eta^{n}(x)\right\}$ is increasing and bounded above by $U$, it must have a limit, call it $M$. We claim that $M=U$; to see this, suppose otherwise that $M<U$. Since $\eta$ is continuous, we have that

$$
M=\lim _{n \rightarrow \infty} \eta^{n}(x)=\eta\left(\lim _{n \rightarrow \infty} \eta^{n-1}(x)\right)=\eta(M),
$$

but this contradicts the assumption that $\eta(x)>x$ for all $x<U$. Therefore, we conclude that $\eta^{n}(x) \rightarrow U$ for each $x<U$.

It will be useful to define the truncated growth subkernel $g_{0}(y, x)$, where

$$
g_{0}(y, x):=\left\{\begin{array}{l}
g(y, x), x \in[L, U), y \geq \eta(x) \\
0, \quad \text { else }
\end{array}\right.
$$

Also, define $G_{0}: L^{1} \rightarrow L^{1}$ to be the integral operator with kernel $g_{0}(y, x)$. We immediately have that $G_{0} \leq G$.

Lemma 5.4 For any $\varphi \in K \backslash\{0\}$, the function $G_{0} \varphi$ is not the zero-function.

Proof We prove the contrapositive, so suppose that $G_{0} \varphi=0$, for some $\varphi \in K$. Then

$$
\begin{aligned}
0 & =\left\|G_{0} \varphi\right\|_{1} \\
& =\int_{L}^{U} \int_{L}^{U} g_{0}(y, x) \varphi(x) d x d y \\
& =\int_{L}^{U} \varphi(x) \int_{L}^{U} g_{0}(y, x) d y d x \\
& =\int_{L}^{U} \varphi(x) \int_{\eta(x)}^{U} g(y, x) d y d x
\end{aligned}
$$

which implies that $\varphi \equiv 0$ a.e., since we have assumed that $\int_{\eta(x)}^{U} g(y, x) d y>0$ for a.e. $x \in[L, U)$.

Corollary 5.3 For any $k \geq 1, G_{0}^{k} \varphi$ is not the zero function. 
Lemma 5.5 Suppose that $\|\varphi\|_{1}>0$. Then for every $n \in \mathbb{N}$,

$$
\int_{\eta^{n}(L)}^{U}\left(G_{0}^{n} \varphi\right)(y) d y>0 .
$$

Proof We proceed by induction. By Lemma 5.4, we know that $G_{0} \varphi$ is nonzero, so $\left\|G_{0} \varphi\right\|_{1}>0$. By definition of the kernel $g_{0}$, we have that $g_{0}(y, x) \equiv 0$ for $x \in[L, U)$ and $y<\eta(L)$, and thus we have

$$
0<\int_{L}^{U}\left(G_{0} \varphi\right)(y) d y=\int_{\eta(L)}^{U}\left(G_{0} \varphi\right)(y) d y
$$

so the base-case holds.

Next, suppose that

$$
\int_{\eta^{k}(L)}^{U}\left(G_{0}^{k} \varphi\right)(y) d y>0
$$

for some $k$. Then, for the sake of a contradiction, suppose that

$$
\begin{aligned}
0 & =\int_{\eta^{k+1}(L)}^{U}\left(G_{0}^{k+1} \varphi\right)(y) d y \\
& =\int_{\eta^{n+1}(L)}^{U} \int_{L}^{U} g_{0}(y, x)\left(G_{0}^{k} \varphi\right)(x) d x d y \\
& =\int_{L}^{U}\left(G_{0}^{k} \varphi\right)(x)\left(\int_{\eta^{n+1}(L)}^{U} g_{0}(y, x) d y\right) d x
\end{aligned}
$$

Assumption (M) implies that $\int_{\eta(x)}^{U} g_{0}(y, x) d y>0$ for a.e $x \in[L, U)$. This implies that

$$
0<\int_{\eta^{n+1}(L)}^{U} g_{0}(y, x) d y=\int_{\eta\left(\eta^{n}(L)\right)}^{U} g_{0}(y, x) d y
$$

for a.e. $x$ in the interval $\left(\eta^{n}(L), U\right)$. Comparison of this with (5.1) yields that $G_{0}^{k} \varphi=0$ on the interval $\left(\eta^{n}(L), U\right)$, but this contradicts the induction hypothesis. Therefore,

$$
0<\int_{\eta^{n}(L)}^{U}\left(G_{0}^{n} \varphi\right)(y) d y
$$

for every $n \in \mathbb{N}$.

Corollary 5.4 For any nonzero $\varphi \in K$ and $\varepsilon>0$, there is an $N \in \mathbb{N}$ such that for $n \geq N, G^{n} \varphi$ is positive on a subset of positive measure in the interval $[U-\varepsilon, U]$. 
Proof Fix $\varepsilon>0$, and let $N \in \mathbb{N}$ be the integer guaranteed by Lemma 5.3 such that $\eta^{n}(L)>U-\varepsilon$ for every $n \geq N$. Applying the result of Lemma 5.5, we have

$$
\int_{U-\varepsilon}^{U}\left(G^{n} \varphi\right)(y) d y \geq \int_{\eta^{n}(L)}^{U}\left(G^{n} \varphi\right)(y) d y \geq \int_{\eta^{n}(L)}^{U}\left(G_{0}^{n} \varphi\right)(y) d y>0 .
$$

Therefore, $G^{n} \varphi>0$ on a subset of positive measure in the interval $[U-\varepsilon, U]$ for any $n \geq N$.

\section{Each operator $T^{k}$ is not compact}

We now move on to showing that all powers of the operator $T$, with growth kernel $g(y, x)$ zero below the diagonal, fail to be compact. This is in contrast to the case with a bounded kernel considered by Ellner and Rees (2006). In fact, we will prove a stronger statement: every power $T^{k}$ fails to be weakly compact. The main result of this section is:

Theorem 6.1 For the integral operator $T: L^{1} \rightarrow L^{1}$ with kernel given by (3.1) and with $g(y, x)$ zero below the diagonal, the operator $T^{k}$ is not weakly compact for any $k \geq 1$.

Corollary 6.1 The operator $T^{k}: L^{1} \rightarrow L^{1}$ is not compact for any $k \geq 1$.

To prove Theorem 6.1, we use the fact that weak compactness and weak sequential compactness are equivalent in Banach spaces. This is known as the Eberlein-Šmulian theorem, and is Theorem V.6.1 in the book by Dunford and Schwartz (1958):

Theorem 6.2 (Eberlein-Šmulian) Let $X$ be a Banach space. Then the following are equivalent:

(1) $X$ is weakly compact,

(2) $X$ is weakly sequentially compact, and

(9) $X$ is weakly limit-point compact.

We will use the following characterization of weakly sequentially compact sets in $L^{1}$, which is Theorem IV.8.11 in the book by Dunford and Schwartz (1958):

Theorem 6.3 If the family $\mathscr{F} \subseteq L^{1}(S, \sigma, \mu)$ is weakly sequentially compact, then

$$
\lim _{\mu(E) \rightarrow 0} \int_{E} h(s) d \mu=0
$$

uniformly for $h \in \mathscr{F}$. If $\mu(S)<\infty$, then this condition is sufficient for $\mathscr{F}$ to be weakly sequentially compact.

For our purposes, the set $S$ is the closed and bounded interval $\Omega=[L, U] \subseteq \mathbb{R}$, which has finite Lebesgue measure; hence, condition (6.1) is necessary and sufficient to determine weakly sequentially compact collections of $L^{1}(\Omega)$.

We now have all the ingredients needed to prove the main theorem of this section: 
Proof of Theorem 6.1 Let $\mathscr{U} \subseteq L^{1}$ be the closed unit ball. Fix $k \geq 1$, and define $\mathscr{F}:=T^{k}(\mathscr{U}) \subseteq L^{1}$. We note that (6.1) holds for any fixed $h \in \mathscr{F}$; however, we will show that this limit is not uniform on $\mathscr{F}$. To this end, put $\delta_{n}:=\frac{1}{n}(U-L)$ for each $n$, and define $E_{n}:=\left[U-\delta_{n}, U\right]$; then $\mu\left(E_{n}\right) \rightarrow 0$.

Further, define the functions

$$
h_{n}:=\frac{1}{\delta_{n}} \cdot \chi_{E_{n}}(x)
$$

for each $n \geq 1$, where $\chi_{E_{n}}$ is the characteristic function on $E_{n}$. Note that

$$
\left\|h_{n}\right\|_{1}=\frac{1}{\delta_{n}} \int_{L}^{U} \chi_{E_{n}}(x) d x=\frac{1}{\delta_{n}} \int_{U-\delta_{n}}^{U} d x=\frac{1}{\delta_{n}} \cdot \delta_{n}=1,
$$

for each $n$. Hence, each $h_{n} \in \mathscr{U}$ and thus $T^{k} h_{n} \in \mathscr{F}$. Also, Corollary 5.1 implies that $\left\|G^{k} h_{n}\right\|_{1}=1$, for each $n$.

By assumption on $s(x)$, there is an $s_{0}$ such that $0<s_{0} \leq s(x)$ for all $x \in[L, U]$. We thus have the lower bound

$$
\int_{E_{n}}\left(T^{k} h_{n}\right)(y) d y \geq s_{0}^{k} \int_{E_{n}}\left(G^{k} h_{n}\right)(y) d y=s_{0}^{k} \cdot\left\|G^{k} h_{n}\right\|_{1}=s_{0}^{k}>0 .
$$

This implies that the limit (6.1) is not uniform on the set $\mathscr{F}$. The contrapositive of Theorem 6.3 gives that the collection $\mathscr{F}$ is not weakly sequentially compact, and the contrapositive of Eberlein-Šmulian implies that that $\mathscr{F}$ is not weakly compact. Therefore, $T^{k}$ fails to be a weakly compact operator for any $k$, since the choice of $k$ was arbitrary.

We note here that the growth operator $G$ is what makes $T^{k}$ non-compact. By a similar argument as in the previous proof, one can show that the limit (6.1) is not uniform on the set $G^{k}(\mathscr{U})$ for any $k \geq 1$.

Theorem 6.1 and its corollary show that neither the Krein-Rutman theorem, nor its most direct generalization (see Theorem 9.4 in the book by Krasnosel'skij et al. (1989)) guarantee that $T: L^{1} \rightarrow L^{1}$ has a positive eigenvector corresponding to its spectral radius.

However, all is not lost: the operator $T$ does have an eigenvector corresponding to its spectral radius, which we prove in Sect. 9.1. Before we will be able to do that, we will need to show that $T$ is strictly nonsupporting, and that $\lambda=r(T)$ is a pole of the resolvent $R(z, T)$.

\section{The operator $T$ is strictly nonsupporting}

Our goal in this section will be to prove that the IPM operator $T$ is strictly nonsupporting in the sense of Sawashima (1964).

We will be able to prove something stronger: for the IPM operator $T$, the integer $p$ in Definition 4.6 will actually be independent of the choice of the nonzero $\varphi \in K$. 
Also, since we only consider the case when $X=L^{1}$, showing that $T^{n} \varphi>0$ almosteverywhere will be sufficient to showing that $T$ is strictly nonsupporting. Hence, the main theorem of this section is:

Theorem 7.1 Suppose that the operator $T=G S+b F$ satisfies the assumptions $(\boldsymbol{M})$ and $(\boldsymbol{R})$. Then there is a $p \in \mathbb{N}$ such that for every nonzero $\varphi \in K \subseteq L^{1}$ and $n \geq p$, the element $T^{n} \varphi$ is positive almost everywhere in $\Omega$.

From this, we get the quick corollary:

\section{Corollary 7.1 The operator $T$ is strictly nonsupporting.}

Proof Let $p \in \mathbb{N}$ denote the integer guaranteed by Theorem 7.1, and take any nonzero elements $\varphi \in K, \varphi^{*} \in K^{*}$. Then $\varphi^{*}$ acts on elements of $L^{1}$ by integration, and also $\int_{L}^{U} \varphi^{*}(t) d t>0$, since $\varphi^{*}$ is nonzero. Fix some $n \geq p$, then we have

$$
\left\langle T^{n} \varphi, \varphi^{*}\right\rangle=\int_{L}^{U}\left(T^{n} \varphi\right)(t) \varphi^{*}(t) d t>0
$$

since $T^{n} \varphi$ is positive almost-everywhere, and $\varphi$ is positive on a set of positive measure. Therefore, $T$ is strictly nonsupporting since the nonzero functions $\varphi, \varphi^{*}$ were arbitrary, and so was the choice of $n \geq p$.

To prove Theorem 7.1, we will first give some lemmas:

Lemma 7.1 Suppose $\varphi(x)>0$ almost-everywhere in $[L, \hat{x}]$. Then $(G \varphi)(y)>0$ for almost-every $y \in[L, \eta(\hat{x})]$.

Proof Since we only need to prove the statement for a.e. $y \in[L, \eta(\hat{x})]$, we can assume without loss of generality that $y \in[L, \eta(\hat{x})]$ satisfies the inequalities (3.11)-(3.12).

First, suppose that $y \in(L, \eta(\hat{x}))$; in this case, assumption (3.12) implies that $g(y, x)>0$ for $(y, x) \in E:=\{y\} \times[L, \hat{x}]$. Thus, $g(y, x) \varphi(x)>0$ on $E$ as well, so

$$
(G \varphi)(y)=\int_{L}^{U} g(y, x) \varphi(x) d x \geq \int_{L}^{\hat{x}} g(y, x) \varphi(x) d x>0,
$$

as claimed.

Next, suppose that $y \in[\eta(L), \eta(\hat{x})]$; in this case, assumption (3.11) says that

$$
\int_{L}^{\eta^{-1}(y)} g(y, x) d x>0 .
$$

This implies that $g(y, x)>0$ on some subset of positive measure contained in $\{y\} \times$ $\left[L, \eta^{-1}(y)\right]$. Note also that $\eta^{-1}(y)<\hat{x}$ since $\eta$ is strictly increasing, so $\varphi(x)>0$ for a.e. $x \in\left[L, \eta^{-1}(y)\right]$. Then we have

$$
(G \varphi)(y)=\int_{L}^{U} g(y, x) \varphi(x) d x \geq \int_{L}^{\eta^{-1}(y)} g(y, x) \varphi(x) d x>0,
$$

as claimed. 
From this, we get the corollary:

Corollary 7.2 If $\varphi(x)>0$ on $[L, \hat{x}]$, with $\hat{x}$ as in the above lemma, then $G^{k} \varphi>0$ almost-everywhere on $\left[L, \eta^{k}(\hat{x})\right]$.

The proof of this is immediate, but we also get:

Corollary 7.3 Suppose $\varphi(x)>0$ almost-everywhere on $[L, \hat{x}]$. Then for any $\hat{y} \in$ $[L, U)$, there is an $N \in \mathbb{N}$ such that $G^{n} \varphi$ is positive almost-everywhere on $[L, \hat{y}]$ for all $n \geq N$.

Proof Fix $\hat{y} \in(L, U)$. From Lemma 5.3, we know that $\eta^{n}(x) \rightarrow U$ for any $x \in$ $[L, U]$. Thus, there is an $N=N(\hat{x})$ such that $\eta^{n}(\hat{x})>\hat{y}$ for all $n \geq N$. Since $\eta(\hat{x})>\hat{x}$ and $\eta$ is assumed to be strictly increasing, we have that

$$
[L, \hat{y}] \subseteq\left[L, \eta^{N}(\hat{x})\right] \subseteq\left[L, \eta^{n}(\hat{x})\right]
$$

for all $n \geq N$. Corollary 7.2 says that $G^{n} \varphi>0$ almost-everywhere on $\left[L, \eta^{n}(\hat{x})\right]$, so we conclude that $G^{n} \varphi>0$ almost-everywhere on $[L, \hat{y}]$ as well, for all $n \geq N$.

Note that both Corollaries 7.2 and 7.3 are still true when applied to the operator $G S$ in place of $G$, since $s(x)$ is positive almost-everywhere. With these facts, we can now prove the main theorem of this section:

Proof of Theorem 7.1 Fix some nonzero $\varphi_{0} \in K$; then there is an $x_{0}<U$ such that $\varphi_{0}(x)>0$ on a subset of positive measure in $\left[L, x_{0}\right]$. Corollary 5.4 implies that there is some $N_{0} \in \mathbb{N}$ such that for $n \geq N_{0}, G^{n} \varphi_{0}>0$ on a subset of positive measure in $\left[x^{\prime}, U\right]$, where $x^{\prime}$ is the "size of maturity" from assumption (3.4). In particular, we have that $F\left((G S){ }^{N_{0}} \varphi_{0}\right)>0$, so

$$
\varphi_{1}=\left(T^{N_{0}+1} \varphi_{0}\right)(x) \geq b(x) F\left((G S)^{N_{0}} \varphi_{0}\right)>0
$$

for almost every $x \in\left[L, x_{b}\right]$, where $x_{b}$ is the maximum offspring size given in assumption (3.3).

Next, choose some $y$ with $U-\varepsilon_{1}<y<U$, where $\varepsilon_{1}>0$ is the value from assumption (R). Using Lemma 5.3, choose $N_{1} \in \mathbb{N}$ such that $\eta^{n}\left(x_{b}\right)>y$ for each $n \geq N_{1}$. Then we have two cases to consider:

Case 1 Suppose $x \in\left[L, U-\varepsilon_{1}\right]$. Then Corollary 7.2 implies that

$$
\left(T^{n} \varphi_{1}\right)(x) \geq\left((G S)^{n} \varphi_{1}\right)(x)>0,
$$

except possibly on a set of measure zero.

Case 2 Suppose $x \in\left(U-\varepsilon_{1}, U\right]$. Note that $(G S)^{n-1} \varphi_{1}$ is positive almost everywhere on $[L, y]$ by the choice of $N_{1}$; then for $n \geq N_{1}+1$, assumption $(\mathbf{R})$ guarantees that

$$
\begin{aligned}
\left(T^{n} \varphi_{1}\right)(x) & \geq\left((G S)^{n} \varphi_{1}\right)(x) \\
& =\left(G S\left(\left(G S^{n-1}\right) \varphi_{1}\right)\right)(x)
\end{aligned}
$$




$$
\begin{aligned}
& =\int_{L}^{U} g(x, t) s(t)\left((G S)^{n-1} \varphi_{1}\right)(t) d t \\
& \geq s_{0}^{n} \int_{t_{1}}^{t_{2}} g(x, t)\left(G^{n-1} \varphi_{1}\right)(t) d t \\
& >0
\end{aligned}
$$

except possibly on a set of measure zero, since $g(x, t)>0$ for almost-every $(x, t) \in$ $\left[U-\varepsilon_{1}, U\right] \times\left[t_{1}, t_{2}\right]$, and because $\left(G^{n-1} \varphi_{1}\right)$ is positive almost-everywhere on $\left[t_{1}, t_{2}\right]$, as $t_{1}, t_{2}<y$.

Therefore, for $n \geq N_{2}:=N_{0}+N_{1}+1$, we have that $T^{n} \varphi_{0}$ is positive almost everywhere in $[L, U]$, which proves the claim since $\varphi_{0} \in K$ was arbitrary and nonzero.

\section{The spectral radius $r(T)$ is a pole of the resolvent $R(z, T)$}

Now that we have proved the operator $T$ is strictly nonsupporting, we move on to proving that $\lambda=r(T)$ is a pole of the resolvent $R(z, T)$; for complex analysis terminology, we follow the book by Rudin (1987). For clarity, we give a short overview of this section: in Lemmas 8.3-8.6 and the intervening corollaries, we compute the spectral radius $r(G S)$, and the essential spectral radii $r_{e}(G S), r_{e}(T)$ explicitly; it turns out that these three values are all equal. Lemmas 8.7-8.9 and Corollary 8.2 serve to show that $\sigma(T)$ contains a value larger than $r_{e}(T)$; this implies that $\lambda=r(T)>r_{e}(T)$, so $\lambda$ is not an element of the essential spectrum $\sigma_{e}(T)$. The remaining results in this section demonstrate that $\lambda$ is indeed a pole of the resolvent $R(z, T)$.

We begin with a lemma about the MNC $\beta$, which follows from properties listed in Definition 4.2.

Lemma 8.1 Let $X$ be a topological vector space, and suppose $V, W \subseteq X$ with $W$ pre-compact; then $\beta(V+W)=\beta(V)$.

Proof Let $V, W \subseteq X$ be as above, where $X$ is some topological vector space. Properties (1) and (2) in Definition 4.2 imply that

$$
\beta(V+W) \leq \beta(V)+\beta(W)=\beta(V),
$$

because $W$ is pre-compact. Since $V \subseteq V+W$, Property (3) implies that

$$
\beta(V) \leq \beta(V+W)
$$

Hence,

$$
\beta(V) \leq \beta(V+W) \leq \beta(V),
$$

and we conclude that $\beta(V)=\beta(V+W)$. 
The following fact about the IPM operator $T: L^{1} \rightarrow L^{1}$ is a result of Proposition 1 given by Schaefer (1960):

Lemma 8.2 The spectral radius $\lambda=r(T)$ is an element of the spectrum $\sigma(T)$.

We now turn our attention to the growth operator $G: L^{1} \rightarrow L^{1}$. The following lemmas are interesting because they demonstrate that the assumption (3.5) allows us to compute upper bounds for $\beta\left(G^{n}\right)$, whereas (3.6) allows us to compute lower bounds. We again denote $\mathscr{U} \subseteq L^{1}$ to be the closed unit ball, and we extend the functions under consideration from their domain $\Omega=[L, U]$ to all of $\mathbb{R}$ by making them identically equal to zero outside $\Omega$.

Lemma 8.3 Suppose $G: L^{1} \rightarrow L^{1}$ satisfies (3.5). Then for all $k \geq 1$, we have that

$$
\beta\left(G^{k}\right):=\beta\left(G^{k}(\mathscr{U})\right) \leq 1,
$$

with equality when $g(y, x)$ satisfies (3.6).

Proof Fix $k \geq 1$, and fix $\delta, \tau$, and $\varphi$ such that, $0<\tau \leq \delta$, and $\varphi \in G^{k}(\mathscr{U})$. Then there is a $\psi \in \mathscr{U}$ such that

$$
\begin{aligned}
& \varphi(t)=\int_{-\infty}^{\infty} g(t, x)\left(G^{k-1} \psi\right)(x) d x \\
& \varphi_{\tau}(t):=\varphi(t+\tau)=\int_{-\infty}^{\infty} g(t+\tau, x)\left(G^{k-1} \psi\right)(x) d x .
\end{aligned}
$$

Also, Corollary 5.1 implies that $\left\|G^{n} \psi\right\|_{1}=1$ for all $n$, in particular for $n=k$ and $n=k-1$. Of course, in the case of $k=1$, this is merely saying that $\left\|G^{0} \psi\right\|_{1}=$ $\|\psi\|_{1}=1$. Then

$$
\begin{aligned}
\left\|\varphi-\varphi_{\tau}\right\|_{1} & =\int_{-\infty}^{\infty}\left|\int_{-\infty}^{\infty}(g(t, x)-g(t+\tau, x))\left(G^{k-1} \psi\right)(x) d x\right| d t \\
& \leq \int_{-\infty}^{\infty}\left|\left(G^{k-1} \psi\right)(x)\right| \int_{-\infty}^{\infty}|g(t, x)-g(t+\tau, x)| d t d x \\
& \leq \int_{-\infty}^{\infty}\left|\left(G^{k-1} \psi\right)(x)\right|\left(\int_{-\infty}^{\infty}|g(t, x)| d t+\int_{-\infty}^{\infty}|g(t+\tau, x)| d t\right) d x \\
& =2 \cdot \int_{-\infty}^{\infty}\left|\left(G^{k-1} \psi\right)(x)\right| d x \\
& =2 .
\end{aligned}
$$

Applying formula (4.7), we conclude that

$$
\beta\left(G^{k}\right)=\frac{1}{2} \lim _{\delta \rightarrow 0} \sup _{\varphi \in G(\mathscr{U})} \sup _{0<\tau \leq \delta}\left\|\varphi-\varphi_{\tau}\right\| \leq 1,
$$


since $\delta, \tau \leq \delta$, and $\varphi$ chosen above were arbitrary. This proves the first part of the claim.

To prove the second part, suppose that $g(y, x)=0$ when $y \leq x$; we will show that $1 \leq \beta(G)$. To this end, fix $\delta>0$ and define the function

$$
\varphi(x):=\frac{1}{\delta} \cdot \chi_{E_{\delta}}(x)
$$

where $\chi_{E}$ is the indicator function on $E$, and $E_{\delta}:=[U-\delta, U]$. Then $\|\varphi\|_{1}=1$, and also $\left\|G^{k} \varphi\right\|_{1}=\left\|\left(G^{k} \varphi\right)_{\tau}\right\|_{1}=1$ by Corollary 5.1, where $\left(G^{k} \varphi\right)_{\tau}$ for $0<\tau \leq \delta$ is the translated function in (4.7).

By Corollary 5.2, the support of the function $G^{k} \varphi$ is a subset of $E_{\delta}$, and the support of the translate $\left(G^{k} \varphi\right)_{\tau}$ is a subset of $[U-\delta-\tau, U-\tau]$. Thus, for $\tau=\delta, G^{k} \varphi$ and $\left(G^{k} \varphi\right)_{\tau}$ have disjoint supports. This means that the quantity $\left\|G^{k} \varphi-\left(G^{k} \varphi\right)_{\tau}\right\|_{1}$ is maximized when $\tau=\delta$, in which case

$$
\left\|G^{k} \varphi-\left(G^{k} \varphi\right)_{\tau}\right\|_{1}=\left\|G^{k} \varphi\right\|_{1}+\left\|\left(G^{k} \varphi\right)_{\tau}\right\|_{1}=2
$$

Hence,

$$
1 \leq \frac{1}{2} \lim _{\delta \rightarrow 0} \sup _{\varphi \in \mathscr{U}} \sup _{0<\tau \leq \delta}\left\|G^{k} \varphi-\left(G^{k} \varphi\right)_{\tau}\right\|_{1}=\beta\left(G^{k}\right) .
$$

Therefore, $\beta\left(G^{k}\right)=1$ whenever $g(y, x)=0$ below the diagonal, since $\beta\left(G^{k}\right) \leq 1$ as well.

Lemma 8.3 is an interesting addition to the result that $G^{k}$ fails to be compact whenever $g(y, x)$ is zero below the diagonal. One can show that $\beta(\mathscr{U})=1$, and Lemma 8.3 shows that for every $k$, the set $G^{k}(\mathscr{U})$ is just as "non-compact" as $\mathscr{U}$. that

In the following lemmas, we consider the growth and survival operator $G S$. Recall

$$
s_{1}:=\sup _{x \in \Omega}\{s(x)\}=s(U) .
$$

Lemma 8.4 For the operator $G S: L^{1} \rightarrow L^{1}$, we have

$$
\beta\left((G S)^{k}\right) \leq s_{1}^{k}
$$

with equality holding when $g(y, x)$ satisfies (3.6).

Proof Note that $s(x) \leq s_{1}$ by assumption, which implies that $(G S)^{k}(\mathscr{U}) \subseteq s_{1}^{k} G(\mathscr{U})$ for all $k$. Properties (3) and (4) of $\beta$ given above imply that

$$
\beta\left((G S)^{k}\right) \leq \beta\left(s_{1}^{k} G^{k}\right) \leq s_{1}^{k} \beta\left(G^{k}\right) \leq s_{1}^{k},
$$

which proves the first claim. 
To show the second claim, we will show that $s_{1} \leq \beta\left((G S)^{k}\right)$; to this end, fix $k \geq 1$ and $\delta>0$. Let $\varphi$ and $E_{\delta}$ be as in Lemma 8.3. For notational convenience, put $\psi:=(G S)^{k} \varphi$. Recall that $\psi(y)=0$ for $y<U-\delta$, and that $\|\psi\|_{1}=1$. Letting $\psi_{\tau}$ denote the $\tau$-translate of $\psi$, we have that the expression $\left\|\psi-\psi_{\tau}\right\|_{1}$ is maximized when $\tau=\delta$, since in this case $\psi$ and $\psi_{\tau}$ have disjoint supports. Then we have

$$
\sup _{0<\tau \leq \delta}\left\|\psi-\psi_{\tau}\right\|_{1}=\left\|\psi-\psi_{\delta}\right\|_{1}=\|\psi\|_{1}+\left\|\psi_{\delta}\right\|_{1}=2\left\|(G S)^{k} \varphi\right\|_{1} \geq 2 s(U-\delta)^{k}
$$

where the inequality comes from the fact that $s(x)$ is increasing. Since we can define such a $\varphi$ for any choice of $\delta>0$, and because $s(x)$ is continuous, we conclude that

$$
\beta\left((G S)^{k}\right)=\frac{1}{2} \lim _{\delta \rightarrow 0} \sup _{\varphi \in \mathscr{U}} \sup _{0<\tau \leq \delta}\left\|\psi-\psi_{\tau}\right\|_{1} \geq \frac{1}{2} \lim _{\delta \rightarrow 0} 2 s(U-\delta)^{k}=s_{1}^{k},
$$

which proves the second claim.

This result allows us to easily compute the essential spectral radius $r_{e}(G S)$ :

Corollary 8.1 The essential spectral radius of $G S$ satisfies the bound $r_{e}(G S) \leq s_{1}$, with equality when $g(y, x)$ satisfies (3.6).

Proof Combining the first result in Lemma 8.4 with formula (4.8) yields

$$
r_{e}(G S)=\lim _{k \rightarrow \infty} \beta\left((G S)^{k}\right)^{1 / k} \leq\left(s_{1}^{k}\right)^{1 / k}=s_{1} .
$$

When $g(y, x)$ is zero below the diagonal, the second result in Lemma 8.4 combined with formula (4.8) yields

$$
s_{1} \leq \lim _{k \rightarrow \infty} \beta\left((G S)^{k}\right)^{1 / k}=r_{e}(G S)
$$

which gives the result.

Our next lemma shows an important relationship between the ordinary spectral radius of $G S$, and its essential spectral radius:

Lemma 8.5 The spectral radius of $G S$ satisfies

$$
r(G S) \leq s_{1},
$$

with equality when $g(y, x)$ satisfies (3.6).

Proof Noting that $\left\|(G S)^{k}\right\|_{1} \leq s_{1}^{k}\left\|G^{k}\right\|_{1}=s_{1}^{k}$ for all $k$, we have by Gelfand's formula (4.5) that $r(G S) \leq s_{1}$, which demonstrates the first claim.

Note that $\sigma_{e}(G S) \subseteq \sigma(G S)$, so necessarily $r_{e}(G S) \leq r(G S)$. When $g(y, x)$ is zero below the diagonal, Corollary 8.1 yields:

$$
s_{1} \leq r_{e}(G S) \leq r(G S) \leq s_{1}
$$

which proves the second claim. 
Lemma 8.6 Let $T=G S+b F$ satisfy (3.6) and (M). Then

$$
r_{e}(T)=r_{e}(G S)=s_{1} \text {. }
$$

Proof Note that $b F: L^{1} \rightarrow L^{1}$ is a compact map since it has finite (1-dimensional) rank. Corollary 4.1 and Corollary 4.11 in the book by Edmunds and Evans (1987), combined with Theorem 8.1 above, imply that

$$
r_{e}(T)=r_{e}(G S+b F)=r_{e}(G S)=s_{1} \text {. }
$$

The next step in showing that $r(T) \in \sigma(T)$ is a pole of $R(z, T)$ is showing that there is some $\mu \in \sigma(T)$ such that $|\mu|>s_{1}=r_{e}(T)$; the following lemmas and corollary accomplish this.

Lemma 8.7 Suppose that $\mu \in \rho(G S)$, the resolvent set of $G S$, and define $\psi:=$ $(\mu I-G S)^{-1} b$. If

$$
F \psi=F(\mu I-G S)^{-1} b=1,
$$

then $\psi$ is an eigenvector for $T$ with eigenvalue $\mu$.

Conversely, if $v$ is an eigenvector for $T$ with eigenvalue $\mu \in \rho(G S)$, then $v$ is in the span of $\psi$, and $F \psi=1$.

Proof Suppose $\mu \in \rho(G S)$, and define $\psi$ as above. Then the condition $F \psi=1$ implies that

$$
\psi=(\mu I-G S)^{-1} b(F \psi)
$$

which can be re-arranged to yield

$$
\mu \psi=G S \psi+b F \psi=T \psi
$$

Hence, $\psi$ is an eigenvector of $T$ with eigenvalue $\mu$.

Conversely, suppose that $v$ is an eigenvector for $T$ with eigenvalue $\mu \in \rho(G S)$. Then we can write

$$
T v=(G S+b F) v=\mu v
$$

which we can re-arrange to get

$$
v=(\mu I-G S)^{-1} b(F v) .
$$

This shows that $v$ is in the span of $(\mu I-G S)^{-1} b$, and also that $F v \neq 0$ since $v$ is an eigenvector. Applying $F$ to both sides of (8.2) and dividing by $F v$, we get

$$
F(\mu I-G S)^{-1} b=1,
$$


which completes the proof.

Lemma 8.8 Let $E:=\left(s_{1}, \infty\right)$, and let $P: E \rightarrow \mathbb{R}$ be given by

$$
P(\mu):=F(\mu I-G S)^{-1} b,
$$

where $g(y, x)$ satisfies (3.6) and (M). Then the following hold:

(i) $P$ is continuous;

(ii) $P$ is strictly decreasing;

(iii) $\lim _{\mu \rightarrow \infty} P(\mu)=0$.

If in addition $s(x)$ satisfies $(\boldsymbol{S})$, then

(iv) $\lim _{\mu \rightarrow s_{1}} P(\mu)=\infty$.

Proof The claim (8.8) follows from the fact that the mapping $\mu \mapsto(\mu I-G S)^{-1}$ is continuous for $\mu$ in the resolvent set of $G S$, and the fact that $F$ is continuous.

Next, we prove (8.8). Take $\mu_{1}, \mu_{2} \in\left(s_{1}, \infty\right)$, such that $\mu_{1}<\mu_{2}$. By Lemma 5.5, there is some $n \geq 1$ such that $(G S)^{n} d>0$ on a subset of positive measure in $\left[x^{\prime}, U\right]$. Recall that $f(x) \geq f_{0}>0$ for $x \in\left[x^{\prime}, U\right]$, and this implies that $F(G S)^{k} d>0$ for any $k \geq n$.

Recall that whenever $\mu>s_{1}=r(G S)$, we can write $(\mu I-G S)^{-1}$ as a Neumann series; thus, we can write $F\left(\mu_{1} I-G S\right)^{-1} b$ as a series of nonnegative terms, and split it into two pieces. The first piece will consist of terms with index less than $n$, which may be 0 , and the second piece, with indices greater than or equal to $n$, will consist exclusively of positive terms. To this end, we have

$$
\begin{aligned}
P\left(\mu_{1}\right) & =F\left(\mu_{1} I-G S\right)^{-1} d \\
& =F\left(\frac{1}{\mu_{1}} \sum_{k=0}^{\infty}\left(\frac{G S}{\mu_{1}}\right)^{k} d\right)=\frac{1}{\mu_{1}} \sum_{k=0}^{n-1} \frac{F(G S)^{k} d}{\mu_{1}^{k}}+\frac{1}{\mu_{1}} \sum_{k=n}^{\infty} \frac{F(G S)^{k} d}{\mu_{1}^{k}} \\
& \geq \frac{1}{\mu_{2}} \sum_{k=0}^{n-1} \frac{F(G S)^{k} d}{\mu_{2}^{k}}+\frac{1}{\mu_{1}} \sum_{k=n}^{\infty} \frac{F(G S)^{k} d}{\mu_{1}^{k}} \\
& >\frac{1}{\mu_{2}} \sum_{k=0}^{n-1} \frac{F(G S)^{k} d}{\mu_{2}^{k}}+\frac{1}{\mu_{2}} \sum_{k=n}^{\infty} \frac{F(G S)^{k} d}{\mu_{2}^{k}}=\frac{1}{\mu_{2}} \sum_{k=0}^{\infty} \frac{F(G S)^{k} d}{\mu_{2}^{k}} \\
& =F\left(\mu_{2} I-G S\right)^{-1} b=P\left(\mu_{2}\right),
\end{aligned}
$$

where the " $\geq$ " line above is a result of $\left(G S^{k}\right) d$ possibly being in the kernel of $F$ when $k \leq n-1$, and the strict inequality comes from the fact $\left(G S^{k}\right) d$ cannot be in the kernel of $F$ for $k \geq n$. Therefore, $P(\mu)$ is strictly decreasing on $\left(s_{1}, \infty\right)$ since $\mu_{1}<\mu_{2}$ implies that $P\left(\mu_{1}\right)>P\left(\mu_{2}\right)$.

To prove (iv), we will show that

$$
\lim _{\mu \rightarrow \infty} F(\mu I-G S)^{-1} \varphi=0
$$


holds for any fixed $\varphi \in L^{1}$ and hence in particular for $\varphi=b$. Recall that the functional $F: L^{1} \rightarrow \mathbb{R}$ and the operator $G S: L^{1} \rightarrow L^{1}$ are bounded, so

$$
\begin{aligned}
\left\|F(\mu I-G S)^{-1} \varphi\right\| & \leq\left\|F(\mu I-G S)^{-1}\right\| \cdot\|\varphi\|_{1}=\left\|\sum_{k=0}^{\infty} \frac{F(G S)^{k}}{\mu^{k+1}}\right\| \cdot\|\varphi\|_{1} \\
& \leq\|F\| \cdot\|\varphi\|_{1} \cdot \sum_{k=0}^{\infty} \frac{\|G S\|^{k}}{\mu^{k+1}}=\|F\| \cdot\|\varphi\|_{1} \cdot \sum_{k=0}^{\infty} \frac{s_{1}^{k}}{\mu^{k+1}} \\
& =\left(\frac{\|F\| \cdot\|\varphi\|_{1}}{\mu}\right) \cdot\left(\frac{1}{1-\frac{s_{1}}{\mu}}\right),
\end{aligned}
$$

and taking the limit $\mu \rightarrow \infty$ yields the result.

Finally, to prove (iv), we will show that

$$
\lim _{\mu \rightarrow s_{1}} F(\mu I-G S)^{-1} \varphi=\infty
$$

holds for any nonzero $\varphi \in K$ and hence (iv) holds in particular for $\varphi=b$. By (S) and an assumption on $f(x)$, there is some $\hat{x}<U$ such that both $s(x)=s_{1}$ and $f(x) \geq f_{0}>0$ almost-everywhere for $x>\hat{x}$.

Using Lemmas 5.3 and 5.5, there is an $N \in \mathbb{N}$ such that the support of $G_{0}^{n} \varphi$ is a subset of positive measure of $[\hat{x}, U]$, and $\left\|G_{0}^{n} \varphi\right\|_{1}>0$ for every $n>N$. Put $m:=\left\|G_{0}^{N} \varphi\right\|_{1}>0$, and $\psi:=G_{0}^{N} \varphi$. Corollary 5.1 now implies that

$$
\left\|G^{k} \psi\right\|_{1}=\|\psi\|_{1}=\left\|G_{0}^{N} \varphi\right\|_{1}=m>0
$$

for all $k \geq 1$.

We now study the nonnegative number

$$
F(\mu-G S)^{-1} \varphi=\frac{1}{\mu} F\left(I-\frac{G S}{\mu}\right)^{-1} \varphi
$$

By splitting the Neumann series for $\left(I-\frac{G S}{\mu}\right)^{-1}$ into terms before $N$ and terms after $N$, we can write

$$
\begin{aligned}
\frac{1}{\mu} F\left(I-\frac{G S}{\mu}\right)^{-1} \varphi & =\frac{1}{\mu} \int_{L}^{U} f(x)\left(\sum_{k=0}^{\infty}\left(\frac{G S}{\mu}\right)^{k} \varphi\right)(x) d x \\
& =M+\frac{1}{\mu} \int_{L}^{U} f(x)\left(\sum_{k=N+1}^{\infty}\left(\frac{G S}{\mu}\right)^{k} \varphi\right)(x) d x
\end{aligned}
$$


where

$$
M:=\frac{1}{\mu} \int_{L}^{U} f(x)\left(\sum_{k=0}^{N}\left(\frac{G S}{\mu}\right)^{k} \varphi\right)(x) d x
$$

is a nonnegative number (and possibly zero). We claim that the right-hand term of (8.4) goes to $\infty$ as $\mu \rightarrow s_{1}$.

Note that the assumption of $g(y, x)$ being zero below the diagonal implies that $\mu^{-1} g(y, x) s(x)$ is also zero below the diagonal; hence, $\left(\frac{G S}{\mu}\right)^{k} \varphi>0$ on a subset of positive measure in $[\hat{x}, U]$ for $k \geq N+1$ by a similar argument to the one given in the proof of Lemma 5.2.

The uniform convergence of the Neumann series allows us to interchange the sum and integral signs in the following calculation:

$$
\begin{aligned}
\frac{1}{\mu} \int_{L}^{U} f(x)\left(\sum_{k=N+1}^{\infty}\left(\frac{G S}{\mu}\right)^{k} \varphi\right)(x) d x & \geq \frac{f_{0}}{\mu} \int_{L}^{U} \sum_{k=N+1}^{\infty}\left(\frac{s_{1}}{\mu}\right)^{k}\left(G^{k} \varphi\right)(x) d x \\
& =\frac{f_{0}}{\mu} \sum_{k=N+1}^{\infty}\left(\frac{s_{1}}{\mu}\right)^{k} \int_{L}^{U}\left(G^{k} \varphi\right)(x) d x \\
& =\frac{f_{0}}{\mu} \sum_{k=N+1}^{\infty}\left(\frac{s_{1}}{\mu}\right)^{k} \underbrace{\left\|G^{k} \varphi\right\|}_{=m, \forall k \geq N+1} \\
& =\frac{f_{0} \cdot m}{\mu}\left(\frac{s_{1}}{\mu}\right)^{N+1} \sum_{k=0}^{\infty}\left(\frac{s_{1}}{\mu}\right)^{k} \\
& =\frac{f_{0} \cdot m}{\mu}\left(\frac{s_{1}}{\mu}\right)^{N+1}\left(\frac{1}{1-\frac{s_{1}}{\mu}}\right)
\end{aligned}
$$

Since $f_{0}, \mu$, and $m$ are positive numbers, taking the limit $\mu \rightarrow s_{1}$ shows that (8.5) goes to $\infty$, which implies that (8.4) also goes to $\infty$. Therefore,

$$
\lim _{\lambda I \rightarrow s_{1}} F(\lambda-G S)^{-1} \varphi=\infty
$$

and in particular for $\varphi=b$.

Corollary 8.2 There exists a unique real-valued $\mu>r_{e}(T)$ such that $P(\mu)=1$.

Proof Recall from Lemma 8.6 that $s_{1}=r_{e}(T)$ when $g(y, x)$ is zero below the diagonal. Then properties (8.8), (8.8), and (iv) of Lemma 8.8 guarantee the existence of such a $\mu$, and property (8.8) guarantees its uniqueness.

Lemma 8.9 If $g(y, x)$ satisfies (3.6), $(\boldsymbol{M})$, and $(\boldsymbol{S})$, then there is an eigenvalue $\mu \in$ $\sigma(T)$ such that $\mu>s_{1}=r_{e}(T)$. 
Proof Corollary 8.1 and Corollary 8.2 guarantee the existence of a unique $\mu>$ $r_{e}(G S)=s_{1}$ such that

$$
P(\mu)=F(\mu I-G S)^{-1} d=1 .
$$

Lemma 8.7 then implies that $\mu$ is an eigenvalue of $T$ with eigenvector $\psi=(\mu I-$ $G S)^{-1} b$; hence, $\mu \in \sigma(T)$ and $\mu>s_{1}$, as claimed.

We note that the purpose of property (iv) of Lemma 8.8 in proving Lemma 8.9 is to show that $P(\hat{\mu})>1$ for some $\hat{\mu}$. If one could verify that $P(\hat{\mu})>1$ for some $\hat{\mu}$ in another way, then assumption (S) would be unnecessary.

The following corollary is a critical result:

Corollary 8.3 Suppose that $g(y, x)$ satisfies (3.6), $(\boldsymbol{M})$, and $(\boldsymbol{S})$, and let $\lambda=r(T)$. Then $\lambda>r_{e}(T)$, which in particular means that $\lambda \in \sigma(T) \backslash \sigma_{e}(T)$.

Proof From Lemma 8.9, we know that there is some $\mu \in \sigma(T)$ such that $\mu>r_{e}(G S)$. Then by the definition of the spectral radius, we have

$$
r(T) \geq \mu>r_{e}(T),
$$

as claimed. This fact, combined with Lemma 8.2, implies that $\lambda \in \sigma(T) \backslash \sigma_{e}(T)$.

The main theorem of this section is now simple to prove:

Theorem 8.1 Suppose that $g(y, x)$ satisfies (3.6), (M), and $(\boldsymbol{S})$. Then the spectral radius $\lambda=r(T)$ is a pole of $R(z)=(z I-T)^{-1}$.

Proof By Corollary 8.3, we know that $\lambda \in \sigma(T) \backslash \sigma_{e}(T)$. The result follows immediately from Theorem A.3.3 in the book by Clément et al. (1987).

\section{Main results}

Now that we have shown the operator $T: L^{1} \rightarrow L^{1}$ is strictly nonsupporting, and that its spectral radius $\lambda=r(T)$ is a pole of its resolvent $R(z, T)$, we can prove that $T$ has the properties (1)-(3) given in the introduction, which we have collected (with even more results) in Theorem 9.1. In the proof, we will make use of results given by Marek (1970) and Sawashima (1964).

Theorem 9.1 Suppose that $T: L^{1} \rightarrow L^{1}$ is an integral operator with kernel of the form (3.1), whose component functions satisfy the assumptions (3.2)-(3.6), (M), (R), and $(\mathrm{S})$. Then $T$ has the following properties:

(1) The spectral radius $\lambda=r(T)$ is positive, and is an eigenvalue for $T$ and $T^{*}$. Moreover, the respective eigenvectors $\psi, \psi^{*}$ span one-dimensional eigenspaces, $\psi$ is quasi-interior, and $\psi^{*}$ represents a strictly positive linear functional. Additionally, $\psi, \psi^{*}$ are the only eigenvectors of $T, T^{*}$ which can be scaled so that $\psi \in K, \psi^{*} \in K^{*}$. 
(2) Thas a spectral gap, meaning that

$$
\sup \{|z| \mid z \in \sigma(T), z \neq \lambda\}<\lambda
$$

(3) Suppose $\psi$ is scaled so that $\|\psi\|_{1}=1$, and $\psi^{*}$ is scaled so that $\left\langle\psi, \psi^{*}\right\rangle=1$. Then for any nonzero $\varphi_{0} \in K$, we have

$$
\lim _{n \rightarrow \infty} \frac{T^{n} \varphi_{0}}{\lambda^{n}}=\left\langle\varphi_{0}, \psi^{*}\right\rangle \psi
$$

where $\left\langle\varphi_{0}, \psi^{*}\right\rangle>0$.

Proof Note that under the above assumptions, $T$ is a strictly nonsupporting operator by Corollary 7.1, and hence in particular is nonsupporting. Also, $\lambda=r(T)$ is a pole of the resolvent $R(z, T)$ by Theorem 8.1. Hence, Theorem 2.3(d) given by Marek (1970) implies that $\lambda$ is the only element of the peripheral spectrum $\sigma_{p}(T)$. Since $\lambda>r_{e}(T)$ by Corollary 8.3 , the value $\lambda$ is not in the essential spectrum. From our definition of the essential spectrum, this means that any eigenspace corresponding to $\lambda$ must be finite-dimensional, so $T$ satisfies the hypotheses of Theorem 5 given by Sawashima (1964), the consequences of which are exactly property (1) above.

Next, we will show that $T$ has a spectral gap; suppose otherwise that

$$
\sup \{|z| \mid z \in \sigma(T), z \neq \lambda\}=\lambda \text {. }
$$

Then there is a sequence $\left\{z_{n}\right\} \subseteq \sigma(T)$ such that $z_{n} \neq \lambda$ for all $n$, and $\left|z_{n}\right| \rightarrow \lambda$. Without loss of generality, we can also assume that

$$
\left|z_{1}\right|<\left|z_{2}\right|<\cdots<\lambda
$$

Then the sequence $\left\{z_{n}\right\}$ is an infinite subset of the closed disc $D \subseteq \mathbb{C}$ of radius $\lambda$, which is a compact set. Theorem 2.37 given by Rudin (1976) says that $\left\{z_{n}\right\}$ must have a limit point in $D$, call it $z_{0}$. Since $\lambda$ is not a limit point of the spectrum, as $\lambda>r_{e}(T)$, it must be that $z_{0} \neq \lambda$. Hence, we must have $\left|z_{0}\right|<\lambda$, and thus $\left|z_{n}\right|<\left|z_{0}\right|<\lambda$ for all $n$. This implies that $\left|z_{n}\right| \nrightarrow \rightarrow \lambda$, contradicting the choice of $\left\{z_{n}\right\}$. We conclude that $T$ must have a spectral gap, namely that

$$
\sup \{|z| \mid z \in \sigma(T), z \neq \lambda\}<\lambda \text {. }
$$

To see that property (3) holds, note that Theorem 2.3(e) given by Marek (1970), says that the operator $B_{1}: L^{1} \rightarrow L^{1}$ defined by

$$
B_{1}:=\lim _{n \rightarrow \infty} \frac{T^{n}}{\lambda^{n}}
$$

is a projection operator onto the eigenspace spanned by $\psi$. Then there is some element $h \in K^{*}$ such that $B_{1} \varphi=\langle\varphi, h\rangle \psi$ for all $\varphi \in L^{1}$; we claim that $h=\psi^{*}$ almost- 
everywhere. To this end, note that

$$
\left\langle\varphi, \psi^{*}\right\rangle \psi=\left\langle\frac{\varphi}{\lambda^{n}}, \lambda^{n} \psi^{*}\right\rangle \psi=\left\langle\frac{\varphi}{\lambda^{n}},\left(T^{*}\right)^{n} \psi^{*}\right\rangle \psi=\left\langle\frac{T^{n} \varphi}{\lambda^{n}}, \psi^{*}\right\rangle \psi
$$

Taking $n \rightarrow \infty$ yields the relation

$$
\left\langle\varphi, \psi^{*}\right\rangle \psi=\lim _{n \rightarrow \infty}\left\langle\frac{T^{n} \varphi}{\lambda^{n}}, \psi^{*}\right\rangle \psi=\left\langle B_{1} \varphi, \psi^{*}\right\rangle \psi,
$$

since the limit in (9.1) is norm-convergence. Thus, for any $\varphi \in L^{1}$ we have

$$
\left\langle\varphi, \psi^{*}\right\rangle=\left\langle B_{1} \varphi, \psi^{*}\right\rangle=\left\langle\langle\varphi, h\rangle \psi, \psi^{*}\right\rangle=\langle\varphi, h\rangle \cdot \underbrace{\left\langle\psi, \psi^{*}\right\rangle}_{=1}=\langle\varphi, h\rangle .
$$

Subtracting the left- and right-hand sides gives $\left\langle\varphi, \psi^{*}-h\right\rangle=0$ for every $\varphi \in L^{1}$, which implies that $\psi^{*}=h$ almost-everywhere, as claimed.

Then for any nonzero $\varphi_{0} \in K$, we have

$$
\lim _{n \rightarrow \infty} \frac{T^{n} \varphi_{0}}{\lambda^{n}}=\left\langle\varphi_{0}, \psi^{*}\right\rangle \psi
$$

where $\left\langle\varphi_{0}, \psi^{*}\right\rangle>0$ since property (1) says that $\psi^{*}$ represents a strictly positive linear functional; this completes the proof.

Additionally, we can give an explicit formula for the leading eigenvector $\psi$ :

Corollary 9.1 Suppose the operator $T: L^{1} \rightarrow L^{1}$ satisfies the assumptions of Theorem 9.1. Then an eigenvector $\psi$ corresponding to $\lambda=r(A)$ is given by the formula

$$
\psi=(\lambda I-G S)^{-1} b
$$

Proof This is an immediate consequence of Lemma 8.7 and Theorem 9.1.

This corollary shows that the $\mu$ from Corollary 8.2 must in fact be $\lambda=r(T)$; we expect this fact to be useful to us in future work, in which we hope to give ways of estimating $\lambda$ for non-compact operators. In the following section, we give a method which can be used to bound $\lambda$ below, but we are not currently aware of a method to approximate $\lambda$ to any desired degree of accuracy.

\section{Estimating $\lambda=r(T)$, the growth rate of the population}

We proved above that for a non-compact operator $T$ considered in this paper, its spectral radius $\lambda=r(T)$ is the asymptotic growth rate of the modeled population, and the associated eigenvector $\psi$ is the stable stage distribution. In order to make IPMs with non-compact operators useful to ecologists, we also need a way to estimate $\lambda$ 
for a given population. For an IPM operator $T$ with a bounded kernel $k(y, x)$, Ellner and Rees (2006) recommend using the midpoint rule for numerical integration to approximate the IPM operator. This is a way of using finite-rank operators (matrices) to approximate $T$, which one can do when $T$ is compact. In this approximation, one gets a sequence $T_{n}$ of finite-rank operators which converge in the operator norm to the compact operator $T$. By a continuity argument, the spectral radii $\lambda_{n}=r\left(T_{n}\right)$ will converge to $\lambda=r(T)$; since we can estimate $\lambda_{n}$ on a computer, we can thus estimate $\lambda$ as well.

However, the operators which we consider in this paper are not compact (see Corollary 6.1). In particular, this means that they cannot be the norm-limit of finite-rank operators. Thus, the approximation method given by Ellner and Rees (2006) may not work to estimate $\lambda=r(T)$. However, we will give a sufficient condition to guarantee $\lambda>1$, using finite-rank approximations, and a monotonicity argument. Thus, this method can be used to show that a given model predicts a growing population.

To this end, define the "truncated" kernel $g_{\varepsilon}(y, x)$ by

$$
g_{\varepsilon}(y, x):= \begin{cases}g(y, x), & (y, x) \in[L, U] \times[L, U-\varepsilon], \\ 0, & \text { else }\end{cases}
$$

which removes the unbounded portion of $g(y, x)$, for some $\varepsilon \in(0, U-L)$. The operator $T_{\varepsilon}: L^{1} \rightarrow L^{1}$ defined by

$$
\left(T_{\varepsilon} \varphi\right)(y):=\int_{L}^{U}\left(s(x) g_{\varepsilon}(y, x)+b(y) f(x)\right) \varphi(x) d x
$$

is compact, and thus its leading eigenvalue $r\left(T_{\varepsilon}\right)$ can be approximated using the methods given by Ellner and Rees (2006). Note that $g_{\varepsilon}(y, x) \leq g(y, x)$ for all $(y, x) \in$ $[L, U)$, so $T_{\varepsilon} \leq T$. Theorem 4.2 in the paper by Marek (1970) then implies that

$$
r\left(T_{\varepsilon}\right) \leq r(T)
$$

This is very useful, because if $r\left(T_{\varepsilon}\right)>1$ for some $\varepsilon$, then (10.2) implies that the the growth rate of the population modeled by $T$ will also be greater than 1 . However, we currently do not know whether $r\left(T_{\varepsilon}\right)$ approximates $r(T)$ as $\varepsilon \rightarrow 0$.

Note also that (10.2) does not help in determining the extinction of the population, which occurs when $r(T)<1$. Until we know whether $r\left(T_{\varepsilon}\right) \rightarrow r(T)$, we cannot rule out the possibility that $r(T)>1$, while also having $r\left(T_{\varepsilon}\right)<1$ for all $\varepsilon$ near 0 .

Lemmens and Nussbaum (2013) studied a similar problem, namely under what conditions $\left\|T_{n}-T\right\|_{K} \rightarrow 0$ implies that $r_{K}\left(T_{n}\right) \rightarrow r_{K}(T)$, where $K$ is a cone,

$$
\|T\|_{K}:=\sup \{\|T x\| ; x \in K \text { and }\|x\| \leq 1\}
$$

and $r_{K}(\cdot)$ denotes the cone spectral radius. We will not define this term here, but instead note that for the operators considered in this paper, the cone spectral radius and ordinary spectral radius coincide; that is, $r_{K}(T)=r(T)$, which is a consequence of Theorem 
2.2 given by Mallet-Paret and Nussbaum (2002). Even in this restricted setting, the condition $\left\|T_{n}-T\right\|_{K} \rightarrow 0$ is not sufficient to guarantee that $r_{K}\left(T_{n}\right) \rightarrow r_{K}(T)$. Lemmens and Nussbaum (2013) give in Section 3 of their paper an example of an operator $\tilde{T}$ compact on a cone $K$, but not on the whole space, and with approximating operators $\tilde{T}_{n}$ which are compact on $K$, such that $\left\|\tilde{T}-\tilde{T}_{n}\right\|_{K} \rightarrow 0$. However, for these particular operators, it turns out that $r_{K}\left(\tilde{T}_{n}\right) \not \nrightarrow r_{K}(\tilde{T})$.

In the case of the IPM operator $T$ and operators $T_{\varepsilon}$ defined in (10.1), it is straightforward to show that $\left\|T-T_{\varepsilon}\right\|_{K} \nrightarrow \supset 0$ as $\varepsilon \rightarrow 0$, so approximating $r(T)$ with $r\left(T_{\varepsilon}\right)$ appears to be an even more difficult problem than the one considered by Lemmens and Nussbaum (2013).

\section{An application to a northern pike population}

In this section, we will show that the results of this paper, combined with the method given in Sect. 10, predict that the growth rate $\lambda$ of an (Esox lucius) population in Windermere lake, England is larger than 1. The IPM we consider here was first used by Vindenes et al. (2014), and was elaborated on by Vindenes and Langangen (2015), Stubberud et al. (2019), and Ohlberger et al. (2020). In modeling the northern pike population, Vindenes et al. (2014) used length as their structure variable. Hence, individuals cannot shrink between time steps. This is the situation of interest to us in this paper, and indeed their growth function $g(y, x)$ is unbounded (see Fig. 1 in Sect. 3). The kernel $k(y, x)$ of their operator $T: L^{1} \rightarrow L^{1}$ given by Vindenes et al. (2014) satisfies assumptions (3.1)-(3.6), in addition to (M), (R), and (S). Hence, all results in this paper apply to $T$, including the fact that no power $T^{k}$ is compact.

Using the component functions given by Vindenes et al. (2014), we investigated the compact operator $T_{\varepsilon}$, defined as in (10.1), in MATLAB. Sampling the kernel $k_{\varepsilon}(y, x)$ with 1000 equally-spaced points on the $x, y$-axes yields a mesh size of

$$
\Delta x=\Delta y=\frac{U-L}{1000}=0.098
$$

and for simplicity we set $\varepsilon=\Delta x$. This generates a $1000 \times 1000$ matrix $M$ which approximates the kernel $k_{\varepsilon}(y, x)$, and a matrix $M \Delta x$ which approximates the operator $T_{\varepsilon}$. Using the eig() function in MATLAB, we estimated $\lambda_{\varepsilon}=r\left(T_{\varepsilon}\right)$, and found that

$$
1.0128 \approx \lambda_{\varepsilon} \leq \lambda
$$

where $\lambda=r(T)$ is the spectral radius of the non-compact IPM operator $T$. Further numerical investigations indicated that the estimated value for $\lambda_{\varepsilon}$ only increases with smaller mesh sizes. We must emphasize that $\lambda_{\varepsilon}$ is not the asymptotic growth rate of the population, because it ignores the contribution of individuals with sizes in the range $[U-\varepsilon, U]$. Instead, $\lambda_{\varepsilon}$ is a lower bound for the true growth rate, which is given by $\lambda$. Since we have the inequality

$$
1<1.0128 \approx \lambda_{\varepsilon} \leq \lambda
$$


this IPM predicts that the growth rate of the northern pike population in Windermere lake is greater than 1 .

\section{Conclusions and future work}

We have shown $T^{k}$ is not compact for any $k \geq 1$, where $T: L^{1} \rightarrow L^{1}$ is an IPM operator which models populations of individuals which do not shrink. A priori, it is unclear whether the operator $T$ will have its spectral radius $\lambda=r(T)$ as an eigenvalue, or that the associated eigenvector $\psi$ is nonnegative, since the theoretical work done on IPMs so far has assumed that the operator is compact. In this paper, we showed that $T$ does possess these properties, namely that $T$ satisfies Properties (1)-(3) given in the introduction. Thus, for populations whose individuals do not shrink, the eigenvalue $\lambda$ is the asymptotic growth rate of the population, and its nonnegative eigenvector $\psi$ is the stable stage distribution.

Assumption (S) is a technical condition we needed to prove Lemma 8.8, and were unable to do so without it. We justified its inclusion in this paper because current IPMs in the literature satisfy it, and because examples of very large individuals are often rare, thus making constant survival near the maximum size a reasonable simplification. However, Vindenes et al. (2014)) expressed reservations about this assumption; they state that in some populations, the survival function $s(x)$ decreases near the maximum size $x=U$, perhaps because of senescence or as an artifact from the method used to monitor the population. Additionally, size-selective harvesting may reduce the survival of larger individuals, for example when anglers prefer large fish. In order to make our results applicable in the largest number of cases, we hope to find more reasonable assumptions to replace assumption $(\mathbf{S})$.

Additionally, there is the practical hurdle of estimating the growth rate $\lambda$; we are unable to use the usual numerical methods suggested by Ellner and Rees (2006) to approximate $r(T)$ and $\psi$, because those methods rely on $T$ having a bounded kernel. In Sect. 10, we gave a method, using only compact operators, to find a lower bound for the spectral radius $\lambda$. However, in the future we hope to give a method which can approximate $\lambda$ to an arbitrary degree of accuracy. We expect Lemma 8.7 to be useful in this regard, because it gives the necessary and sufficient condition

$$
F(\lambda I-G S)^{-1} b=1,
$$

for $\lambda$ to be the leading eigenvalue for $T$. Estimating $\lambda$ then becomes a problem about estimating the zero of the function

$$
Q(t):=F(t I-G S)^{-1} b-1
$$

in the set $\left(s_{1}, \infty\right)$.

Acknowledgements Richard Rebarber and Matt Reichenbach were partially supported by National Science Foundation Division of Mathematical Sciences Grant 141259. Brigitte Tenhumberg was supported by USDA-NIFA Grant 2017-03807, and the U.S. National Science Foundation (DEB 1655117). We would 
like to thank an anonymous reviewer, whose suggestions and constructive comments have greatly improved the quality of this paper.

\section{References}

Aalto E, Micheli F, Boch C, Montes JE, Wilson C, De Leo G (2019) Catastrophic mortality, Allee effects, and marine protected areas. Am Nat 193(3):391-408

Akhmerov R, Kamenskii M, Potapov A, Rodkina A, Sadovskii B (1992) Measures of noncompactness and condensing operators. Birkhäuser Verlag, Boston (translated from the Russian by A. Iacob)

Anselone P, Lee JW (1974) Spectral properties of integral operators with nonnegative kernels. Linear Algebra Appl 9:67-87

Bonsall F (1958) Linear operators in complete positive cones. Proc Lond Math Soc 3(9):54-75

Briggs J, Dabbs K, Holm M, Lubben J, Rebarber R, Tenhumberg B (2010) Structured population dynamics: an introduction to integral modeling. Math Mag 83:243-257

Browder F (1961) On the spectral theory of elliptic differential operators. Math Ann 142:22-130

Bruno J, Ellner S, Vu I, Kim K, Harvell C (2011) Impacts of aspergillosis on sea fan coral demography: modeling a moving target. Ecol Monogr 81(1):123-139

Caswell H (2001) Matrix population models: construction, analysis, and interpretation, 2nd edn. Sinauer Associates, Inc, Sunderland

Childs D, Rees M, Rose K, Grubb P, Ellner S (2003) Evolution of complex flowering strategies: an ageand size-structured integral projection model. Proc R Soc B Biol Sci 270:1829-1838

Childs D, Coulson T, Pemberton J, Clutton-Brock T, Rees M (2011) Predicting trait values and measuring selection in complex life-histories: reproductive allocation decisions in Soay sheep. Ecol Lett 10:985992

Clément P, Heijmans H, Angenent S, van Duijn C, de Pagter B (1987) One-parameter semigroups. Elsevier, Amsterdam

Conway JB (1994) A course in functional analysis, 2nd edn. Springer, New York

Coulson T, MacNulty D, Stahler D, vonHoldt B, Wayne R, Smith D (2011) Modeling effects of environmental change on wolf populations dynamics, trait evolution, and life history. Science 334:1275-1278

Dunford N, Schwartz J (1958) Linear operators, part I: general theory. Interscience, New York

Eager E, Haridas C, Pilson D, Rebarber R, Tenhumberg B (2013) Disturbance frequency and vertical distribution of seeds affect long-term population dynamics: a mechanistic seed bank model. Am Nat 182(2):180-190

Easterling M (1998) The integral projection model: theory, analysis, and application. Ph.D. thesis, North Carolina State University

Edmunds D, Evans W (1987) Spectral theory and differential operators. Oxford University Press, New York

Edmunds D, Potter A, Stuart C (1972) Non-compact positive operators. Proc R Soc Ser A Math Phys Sci 328(1572):67-81

Ellner S, Rees M (2006) Integral projection models for species with complex demography. Am Nat 167(3):410-428

Ellner S, Childs D, Rees M (2016) Data-driven modelling of structured populations: a practical guide to the integral projection model. Springer, Cham

Hegland S, Jongejans E, Rydgren K (2010) Investigating the interaction between ungulate grazing and resource effects on Vaccinium myrtillus populations with integral projection models. Oecologia 163(3):695-706

Jacquemyn H, Brys R, Jongejans E (2010) Size-dependent flowering and costs of reproduction affect population dynamics in a tuberous perennial woodland orchid. J Ecol 98:1204-1215

Karlin S (1959) Positive operators. J Math Mech 8(6):907-937

Krasnosel'skij M, Lifshits J, Sobolev A (1989) Positive linear systems: the method of positive operators. Heldermann Verlag, Berlin (translated from the Russian by Jürgen Appell)

Krein M, Rutman M (1950) Linear operators leaving invariant a cone in a Banach space. Am Math Soc Transl Ser 1(10):199-325

Lemmens B, Nussbaum R (2013) Continuity of the cone spectral radius. Proc Am Math Soc 141(8):27412754 
Lubben J, Boeckner D, Rebarber R, Townley S, Tenhumberg B (2009) Parameterizing the growth-decline boundary for uncertain population projection models. Theor Popul Biol 75:85-97

Mallet-Paret J, Nussbaum R (2002) Eigenvalues for a class of homogeneous cone maps arising from maxplus operators. Discrete Contin Dyn Syst 8(3):519-562

Marek I (1967) $u_{0}$-positive operators and some of their applications. SIAM J Appl Math 15(3):484-494

Marek I (1970) Frobenius theory of positive operators: comparison theorems and applications. SIAM J Appl Math 19(3):607-628

Miller T, Williams J, Jongejans E, Brys R, Jacquemyn H (2012) Evolutionary demography of iteroparous plants: incorporating non-lethal costs of reproduction into integral projection models. Proc R Soc B Biol Sci 279(1739):2831-2840

Niiro F, Sawashima I (1966a) On positive irreducible operators in an arbitrary Banach lattice and a problem of H. H. Schaefer. Proc Jpn Acad 42(7):677-681

Niiro F, Sawashima I (1966b) On the spectral properties of positive irreducible operators in an arbitrary Banach lattice and problems of H. H. Shaefer. Sci Pap Coll Gen Educ Univ Tokyo 15:145-183

Nussbaum R (1970) The radius of the essential spectrum. Duke Math J 38:473-478

Ohlberger J, Langangen $\varnothing$, Winfield I, Vindenes Y (2020) The importance of variation in offspring body size for stability in cannibalistic populations. Oikos 129:59-69

Raghavan T (1965) On linear operators leaving a convex set invariant in Banach spaces. Indian J Stat 10(2):293-302

Rees M, Rose K (2002) Evolution of flowering strategies in Oenothera glazioviana: an integral projection model approach. Proc R Soc B Biol Sci 269(1499):1509-1515

Rudin W (1976) Principles of mathematical analysis, 3rd edn. McGraw Hill, New York

Rudin W (1987) Real and complex analysis, 3rd edn. McGraw-Hill, New York

Sawashima I (1964) On spectral properties of some positive operators. Nat Sci Rep Ochanomizu Univ 15(2):53-64

Schaefer H (1960) Some properties of positive linear operators. Pac J Math 10:1009-1019

Stubberud M, Vindenes Y, Vøllestad L, Winfield I, Stenseth N, Langangen Ø (2019) Effects of size- and sex-selective harvesting: an integral projection model approach. Ecol Evol 9(22):12556-12570

Tenhumberg B, Suwa T, Tyre A, Russell L, Louda S (2015) Integral projection models show exotic thistle is more limited than native thistle by ambient competition and herbivory. Ecosphere 6(4):1-18

Vindenes Y, Langangen $\varnothing$ (2015) Individual heterogeneity in life histories and eco-evolutionary dynamics. Ecol Lett 18:417-432

Vindenes Y, Edeline E, Ohlberger J, Langangen Ø, Winfield I, Stenseth N, Vøllestad L (2014) Effects of climate change on trait-based dynamics of a top predator in freshwater ecosystems. Am Nat 183(2):243-256

Publisher's Note Springer Nature remains neutral with regard to jurisdictional claims in published maps and institutional affiliations. 